\title{
A Facile Synthesis of Pyrrolidine-based Iminosugars as Potential Alpha-Glucosidase Inhibitors
}

\author{
MUHAMAD ZULFAQAR BACHO', MOHD FAZLI MOHAMMAT², ZURINA SHAAMERI², \\ AGUSTONO WIBOWO ${ }^{3}$, FIRDAUS KAMARULZAMAN ${ }^{4}$ and AHMAD SAZALI HAMZAH ${ }^{2 *}$
}

${ }^{1}$ Faculty of Applied Sciences, Universiti Teknologi Mara (UiTM), 40450 Shah Alam, Selangor Darul Ehsan, Malaysia.

${ }^{2}$ Organic Synthesis Laboratory, Institute of Science, Universiti Teknologi MARA (UiTM), 42300 Bandar Puncak Alam, Selangor Darul Ehsan, Malaysia.

${ }^{3}$ Faculty of Applied Sciences, Cawangan Pahang, Universiti Teknologi Mara (UiTM), 26400 Bandar Jengka, Pahang, Malaysia.

${ }^{4}$ Natural Products Division, Forest Research Institute Malaysia (FRIM), Kepong, 52109 Selangor Darul Ehsan, Malaysia.

${ }^{*}$ Corresponding author E-mail: asazali @ uitm.edu.my

http://dx.doi.org/10.13005/ojc/360214

(Received: October 14, 2019; Accepted: April 01, 2020)

\section{ABSTRACT}

A multifaceted approach comprising MCR (multicomponent reaction), amination and stereoselective reduction reactions was used to synthesize new pyrrolidine-based iminosugars. The key step of this strategy involves the contruction of a highly functionalised pyrroldine ring skeleton through MCR approach. Subsequently, amination and reduction reactions to the ring skeleton provide a quick access to new pyrrolidine-based imino sugars. The iminosugars were then tested against alpha glucosidase activity in which one compound (4-((4-methoxyphenyl)amino)pyrrolidin-3-ol), was found to be the most potent at low dosage.

Keywords: Iminosugars, Pyrrolidine, Alpha-glucosidase, Antidiabetic.

\section{INTRODUCTION}

Diabetes mellitus (DM) is a continuously rising chronic metabolic disorder and has become a vital problem to the world population'. Over time, this disease has escalated the number of health issues among patients commonly to the type 2 diabetes $(\mathrm{T} 2 \mathrm{D})^{2}$. T2D happens by an abnormal postprandial increase of blood glucose level due to failed insulin production or action ${ }^{3}$. One way to overcome this calamity is by using alpha-glucosidase inhibitors, an approach to prevent postprandial hyperglycemia since they can act as competitive inhibitors of small intestinal brush-border alpha-glucosidases ${ }^{4,5}$. This glucosidase can inhibits the enzymes and delay the conversion of polysaccharide into absorbable monosaccharides (glucose and fructose) ${ }^{6}$. Some alpha-glucosidases such as acarbose, voglibose

This is an Open Access article licensed under a Creative Commons license: Attribution 4.0 International (CC- BY). Published by Oriental Scientific Publishing Company @ 2018

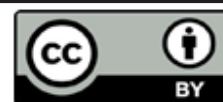


and miglitol that are currently available in the market prove the effectiveness of this approach (Fig. 1) ${ }^{7}$. Due to the limited number of these commercially available inhibitors, new alternative synthetic methods are thus required to combat this disease effectively.

For the past decade, many groups have reported the synthesis of pyrrolidine-based iminosugars ${ }^{8}$. Zhang and co-workers reported the synthesis of iminosugars using D-glucose as it's main precursor. The target molecule (3S,4S)-3-((R)-1-2dihydroxyethyl)pyrrolidine-3,4-diol was obtained in 10 steps with $24 \%$ yield ${ }^{9}$. Doddi and co-workers reported the synthesis of azasugars utilizing pyrrolidine skeleton followed by regiospecific amination, ring closing metathesis, and diastereospecific dihydroxylations as the key reactions. These sugar molecules however, were found to have moderate inhibition against glycosidase enzyme ${ }^{6}$. In addition other synthetic strategies of pyrrolidine-based iminosugars have also been reported ${ }^{10,11,12}$.

In continuation of our work on the five membered heterocycle system ${ }^{7,8,13}$, we now report the synthesis of pyrrolidine based iminosugars in short steps via multi component reaction (MCR), amination, stereoselective reduction.
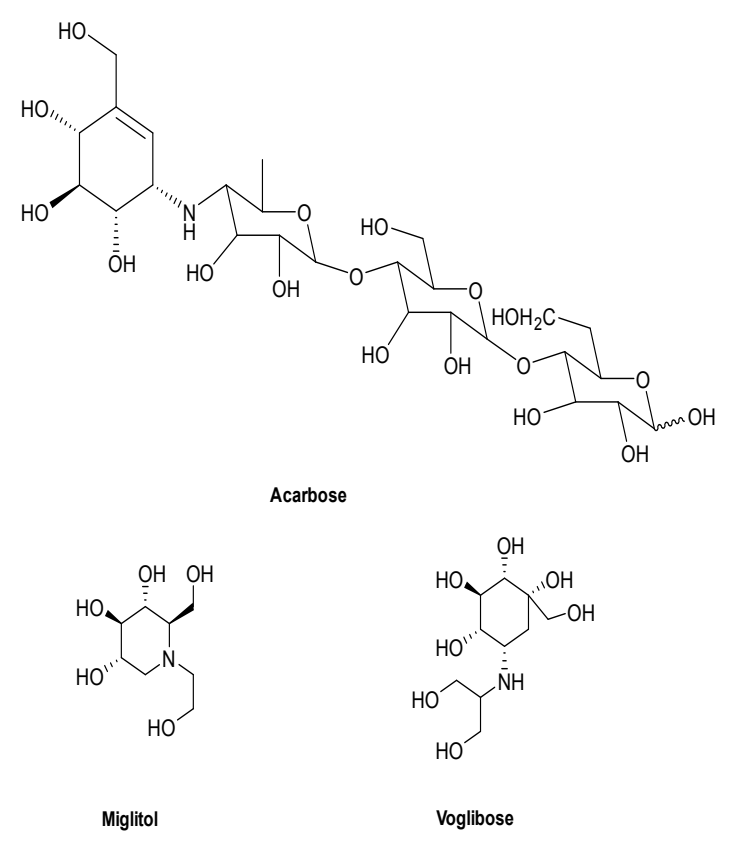

Fig. 1. Structures of some alpha-glucosidase inhibitors

\section{EXPERIMENTAL}

\section{General}

Infrared spectra (IR) was recorded on NICOLET 6700 FT-IR using diamond with ATR. NMR spectra was recorded on JEOL Resonance ECZ400 $\left[400 \mathrm{MHz}\left({ }^{1} \mathrm{H}\right)\right.$ and $\left.100 \mathrm{MHz}\left({ }^{13} \mathrm{C}\right)\right]$ using TMS as the internal standard. The molecular weight of synthesized compound was recorded on Agilent Technnologies model: 6560 Accurate-Mass Q-TOF LC/MS, Thermo Scientific Orbitrap Fusion Tribid Mass Spectrometer and GC-MS Agilent Technologies 7693 Autosampler (GC System). Elemental analysis was performed by Flash 2000 Organic Elemental Analyzer. Melting point was run by Stuart SMP30. Analytical TLC was performed on silica gel 60 F254, Merck (layer thickness $0.25 \mathrm{~mm}$, Merck) and visualized with UV light and $\mathrm{KMnO}_{4}$ as the detecting agent.

\section{General procedure for the synthesis of pyrrolidine- based iminosugar intermediates (4a-k).}

A mixture of compound 1 (1 equiv) and aldehyde (1 equiv) together with an equimolar amount of amine in ethanol was refluxed towards completion ( $0.5-2$ hours). Iced-water was added to the mixture after cooling and $\mathrm{HCl}$ was then added dropwise to $\mathrm{pH} 1$. Filter the solid while appear. Traces aldehyde in the crude product was washed with water and ether to give (4a-k).

\section{Ethyl 4-hydroxy-5-oxo-2,5-dihydro-1H-pyrrole-3- carboxylate (4a)}

White solid;60\%; m.p. 106-109 ${ }^{\circ} \mathrm{C}$. IR (ATR) v/ $\mathrm{cm}^{-1}: 3344(\mathrm{OH}), 2986$ (NH, amide), 1782 (C=O, ester), $1687(\mathrm{C}=\mathrm{C}), 1670$ (-N-C=O, amide), $1302(\mathrm{C}-\mathrm{N}) ;{ }^{1} \mathrm{H}-$ NMR $\left(400 \mathrm{MHz}, \mathrm{CDCl}_{3}\right): \delta 4.91-4.85\left(2 \mathrm{H}, \mathrm{s}, \mathrm{CH}_{2}\right)$, 4.39-4.32 $\left(2 \mathrm{H}, q, \mathrm{~J}=7.2 \mathrm{~Hz}, \mathrm{CH}_{2}\right), 1.38-1.31(3 \mathrm{H}, \mathrm{t}, \mathrm{J}=$ $\left.7.1 \mathrm{~Hz}, \mathrm{CH}_{3}\right) ;{ }^{13} \mathrm{C}-\mathrm{NMR}\left(100 \mathrm{MHz}, \mathrm{CDCl}_{3}\right): \delta 166.54$ $(\mathrm{COH}), 164.23(\mathrm{C}=\mathrm{O}) 151.29(\mathrm{C}=\mathrm{O}), 116.09$ (quat. $\mathrm{C})$, $66.26\left(\mathrm{OCH}_{2}\right), 62.09\left(\mathrm{CH}_{2}\right), 14.34\left(\mathrm{CH}_{3}\right)$; Anal. Calcd. for $\mathrm{C}_{7} \mathrm{H}_{9} \mathrm{NO}_{4}$ : C, 49.12; $\mathrm{H}, 5.30 ; \mathrm{N}, 8.18 ; \mathrm{O}, 37.39$. Found: C, 49.30; H, 4.65; N, 7.04; O, 39.01; GCMS m/z $(\mathrm{El},+\mathrm{ve})$ : found $172.00\left([\mathrm{M}]^{+}\right), \mathrm{C}_{7} \mathrm{H}_{9} \mathrm{NO}_{4}$ calculated 172.06 .

Ethyl 4-hydroxy-1-methyl-5-oxo-2,5-dihydro-1Hpyrrole-3-carboxylate (4b)

Yellowish solid; $40 \%$; m.p. $139-141^{\circ} \mathrm{C}$. IR (ATR) $v / \mathrm{cm}^{-1}: 3400(-\mathrm{OH}), 1780(\mathrm{C}=\mathrm{O}$, ester $), 1648$ $(\mathrm{C}=\mathrm{C}), 1274$ (C-N), 734; ${ }^{1} \mathrm{H}-\mathrm{NMR}\left(400 \mathrm{MHz}, \mathrm{CDCl}_{3}\right.$ ): 
$\delta 1.33\left(3 \mathrm{H}, \mathrm{t}, \mathrm{J}=7.2 \mathrm{~Hz}, \mathrm{CH}_{3}\right), 3.07\left(3 \mathrm{H}, \mathrm{s}, \mathrm{NCH}_{3}\right)$, $3.96\left(2 \mathrm{H}, \mathrm{s}, \mathrm{CH}_{2}\right), 4.31\left(2 \mathrm{H}, q, \mathrm{~J}=7.2 \mathrm{~Hz}, \mathrm{OCH}_{2}\right) ;{ }^{13} \mathrm{C}-$ $\operatorname{NMR}(100 \mathrm{MHz}, \mathrm{CDCl}): \delta 14.30\left(\mathrm{CH}_{3}\right), 30.10\left(\mathrm{NCH}_{3}\right)$, $48.10\left(\mathrm{CH}_{2}\right), 61.20\left(\mathrm{OCH}_{2}\right), 107.60$ (quat. C), 157.40 $(\mathrm{C}=\mathrm{O}), 164.10(\mathrm{C}=\mathrm{O}), 165.20(\mathrm{COH})$; Anal.Calcd. for $\mathrm{C}_{8} \mathrm{H}_{11} \mathrm{NO}_{4}$ (185.07): C, 51.89; $\mathrm{H}, 5.99 ; \mathrm{N}, 7.56 ; \mathrm{O}$, 34.56. Found: $\mathrm{C}, 51.80 ; \mathrm{H}, 4.65 ; \mathrm{N}, 7.70 ; \mathrm{O}, 35.85$.

\section{Ethyl 2-ethyl-4-hydroxy-5-oxo-2,5-dihydro-1H- pyrrole-3-carboxylate (4c)}

White solid; $12.93 \%$; m.p. $146-$ $148^{\circ} \mathrm{C}$. IR (ATR) $v / \mathrm{cm}^{-1}: 3305(\mathrm{OH}), 3176(\mathrm{NH}$, amide), $1766(\mathrm{C}=\mathrm{O}$, ester $), 1678(\mathrm{C}=\mathrm{C}), 1639$ ( $\mathrm{N}-\mathrm{C}=\mathrm{O}$, amide), $1454\left(\mathrm{CH}_{2}\right), 1371\left(\mathrm{CH}_{3}\right), 1304$ (C-N); ${ }^{1} \mathrm{H}$ NMR $\left(400 \mathrm{MHz}, \mathrm{CDCl}_{3}\right): \delta 4.31-4.25$ $(\mathrm{m}, 1 \mathrm{H}), 4.28-4.17(\mathrm{~m}, 2 \mathrm{H}), 2.05-1.90(\mathrm{~m}, 1 \mathrm{H}), 1.66$ $(\mathrm{td}, \mathrm{J}=14.4,7.3 \mathrm{~Hz}, 1 \mathrm{H}), 1.28$ (tdd, $\mathrm{J}=14.9,7.2$, $1.0 \mathrm{~Hz}, 3 \mathrm{H}), 0.85-0.69(\mathrm{~m}, 3 \mathrm{H}) ;{ }^{13} \mathrm{C}$ NMR $(100 \mathrm{MHz}$, $\left.\mathrm{CDCl}_{3}\right): \delta 167.31(\mathrm{COH}), 164.05(\mathrm{C}=\mathrm{O}), 155.28$ $(\mathrm{C}=\mathrm{O}), 112.47$ (quat. $\mathrm{C}$ ), $60.24\left(\mathrm{OCH}_{2}\right), 54.37$ (CH), $24.79\left(\mathrm{CH}_{2}\right), 13.32\left(\mathrm{CH}_{3}\right), 7.04\left(\mathrm{CH}_{3}\right)$; Anal. Calcd. for $\mathrm{C}_{9} \mathrm{H}_{13} \mathrm{NO}_{4}$ : C, 54.26; $\mathrm{H}, 6.58 ; \mathrm{N}, 7.03 ; \mathrm{O}$, 32.13. Found: $\mathrm{C}, 53.69 ; \mathrm{H}, 6.49$; N, 6.91; O, 32.91; GCMS $m / z$ (El,+ve): found 199.00([M] $\left.{ }^{+}\right), \mathrm{C}_{9} \mathrm{H}_{13} \mathrm{NO}_{4}$ calculated 199.08.

\section{Ethyl 4-hydroxy-2-isopropyl-5-oxo-2,5-dihydro- 1H-pyrrole-3-carboxylate (4d)}

White solid; $29.57 \%$; m.p. $165-$ $167^{\circ} \mathrm{C}$. IR (ATR) v/ $\mathrm{cm}^{-1}: 3318(\mathrm{OH}), 3177(\mathrm{NH}$, amide), $2925(\mathrm{C}-\mathrm{H}), 1768$ (C=O, ester), 1722 $(\mathrm{C}=\mathrm{C}), 1630(\mathrm{~N}-\mathrm{C}=\mathrm{O}$, amide $), 1372\left(\mathrm{CH}_{3}\right)$; ${ }^{1} \mathrm{H}-\mathrm{NMR}\left(400 \mathrm{MHz}, \mathrm{CDCl}_{3}\right): \delta 4.31-4.22(\mathrm{~m}, 2 \mathrm{H})$, $4.19(\mathrm{~d}, \mathrm{~J}=2.8 \mathrm{~Hz}, 1 \mathrm{H}), 2.42(\mathrm{dtd}, \mathrm{J}=13.9,7.0,2.8$ $\mathrm{Hz}, 1 \mathrm{H}), 1.30(\mathrm{~s}, 3 \mathrm{H}), 1.07$ (d, J = 7.1 Hz, 3H), 0.59 $(d, J=6.8 \mathrm{~Hz}, 3 \mathrm{H}) ;{ }^{13} \mathrm{C} \mathrm{NMR}\left(100 \mathrm{MHz}, \mathrm{CDCl}_{3}\right): \delta$ $167.85(\mathrm{COH}), 164.13(\mathrm{C}=\mathrm{O}), 155.31(\mathrm{C}=\mathrm{O}), 112.83$ (quat. C), $60.28\left(\mathrm{OCH}_{2}\right), 58.64(\mathrm{CH}), 29.01(\mathrm{CH})$, $19.83\left(\mathrm{CH}_{3}\right), 13.18\left(\mathrm{CH}_{3}\right)$; Anal. Calcd. for $\mathrm{C}_{10} \mathrm{H}_{15} \mathrm{NO}_{4}$ : C, 56.33; H, 7.09; N, 6.57; O, 30.01. Found: C, 55.30; $\mathrm{H}, 6.89 ; \mathrm{N}, 6.17 ; \mathrm{O}, 31.64 ; \mathrm{GCMS} \mathrm{m} / \mathrm{z}(\mathrm{El},+\mathrm{ve})$ : found $213.10\left([\mathrm{M}]^{+}\right), \mathrm{C}_{10} \mathrm{H}_{15} \mathrm{NO}_{4}$ calculated 213.10.

\section{Ethyl 4-hydroxy-5-oxo-2-(p-tolyl)-2,5-dihydro-1H- pyrrole-3-carboxylate (4e)}

Light yellow solid; $28.04 \%$; m.p. $153-156^{\circ} \mathrm{C}$. IR (ATR) v/cm ${ }^{-1}: 3303(\mathrm{OH}), 2982$ (NH, amide), 1719 ( $\mathrm{C}=\mathrm{O}$, ester), $1621(\mathrm{C}=\mathrm{C}) 1556(\mathrm{~N}-\mathrm{C}=\mathrm{O}$, amide), $667\left(\mathrm{Ar}^{-} \mathrm{CH}_{3}\right) ;{ }^{1} \mathrm{H}-\mathrm{NMR}\left(400 \mathrm{MHz}, \mathrm{CDCl}_{3}\right): \delta 7.12$ (s, 2H), 7.07 (s, 2H), 4.34 (q, J=7.2 Hz, 2H), 4.14 (dd, J = 7.1, 1.6 Hz, 1H), $2.31(d, J=9.6 \mathrm{~Hz}, 3 \mathrm{H}$ ), $1.36(\mathrm{t}, \mathrm{J}=7.1 \mathrm{~Hz}, 3 \mathrm{H}) ;{ }^{13} \mathrm{C} \mathrm{NMR}\left(100 \mathrm{MHz}, \mathrm{CDCl}_{3}\right): \delta$ $170.59(\mathrm{COH}), 165.52(\mathrm{C}=\mathrm{O}), 160.36(\mathrm{C}=\mathrm{O}), 140.56$ (aromatic. C), 137.29 (aromatic. C), 129.45 (CH-Ar), $127.14(\mathrm{CH}-\mathrm{Ar}), 107.97$ (quat. C), $64.11\left(\mathrm{OCH}_{2}\right)$, $56.85(\mathrm{CH}), 21.25(\mathrm{CH}-\mathrm{Ar}), 13.89\left(\mathrm{CH}_{3}\right)$; Anal. Calcd. for $\mathrm{C}_{14} \mathrm{H}_{15} \mathrm{NO}_{4} ; \mathrm{C}, 61.85 ; \mathrm{H}, 5.88 ; \mathrm{N}, 4.81 ; \mathrm{O}, 27.46$. Found: C, 55.10; H, 5.12; N, 3.37, O, 36.41; GCMS $\mathrm{m} / \mathrm{z}(\mathrm{El},+\mathrm{ve})$ : found $284.1\left([\mathrm{M}+\mathrm{Na}]^{+}\right), \mathrm{C}_{14} \mathrm{H}_{15} \mathrm{NO}_{4}$ calculated 284.09.

Ethyl 2-(4-cyanophenyl)-4-hydroxy-5-oxo-2,5dihydro-1H-pyrrole-3-carboxylate (4f)

Yellow solid; $20.49 \%$; m.p. $139-141^{\circ} \mathrm{C}$. IR (ATR) v/cm $13302(\mathrm{OH}), 2987$ ( $\mathrm{NH}$, amide), 1728 ( $\mathrm{C}=\mathrm{O}$, ester), $1591(\mathrm{C}=\mathrm{C}), 1501(\mathrm{~N}-\mathrm{C}=\mathrm{O}), 768$ (Ar$\mathrm{CN}) ;{ }^{1} \mathrm{H}-\mathrm{NMR}\left(400 \mathrm{MHz}, \mathrm{CD}_{3} \mathrm{OD}\right): \delta$ 7.76-7.61 (2H), 7.54-7.39 (2H), 5.37-5.22 (1H), 4.12-3.99 (2H), 1.17$1.00(3 \mathrm{H}) ;{ }^{13} \mathrm{C}$ NMR (100 MHz, $\left.\mathrm{CD}_{3} \mathrm{OD}\right): \delta 167.39$ $(\mathrm{COH}), 165.66(\mathrm{C}=\mathrm{O}), 163.10(\mathrm{C}=\mathrm{O}), 143.63$ (quat. C), 132.14 (CH-Ar), 128.29 (CH-Ar), $118.43(\mathrm{CN})$, 111.66 (quat. C), 110.38 (quat. C), $60.67\left(\mathrm{CH}_{2}\right)$, $39.46(\mathrm{CH}), 12.94\left(\mathrm{CH}_{3}\right)$; Anal. Calcd. for $\mathrm{C}_{14} \mathrm{H}_{12} \mathrm{~N}_{2} \mathrm{O}_{4}$; $\mathrm{C}, 61.76 ; \mathrm{H}, 4.44 ; \mathrm{N}, 10.29 ; \mathrm{O}, 23.51$. Found: C, 58.36; $\mathrm{H}, 4.31 ; \mathrm{N}, 6.92 ; \mathrm{O}, 30.41$ : GCMS $\mathrm{m} / \mathrm{z}$ (El, + ve): found $274.2\left([\mathrm{M}+2 \mathrm{H}]^{+}\right), \mathrm{C}_{14} \mathrm{H}_{12} \mathrm{~N}_{2} \mathrm{O}_{4}$ calculated 274.09.

Ethyl 4-hydroxy-2-(4-methoxyphenyl)-1-methyl-5oxo-2,5-dihydro-1 $\mathrm{H}$-pyrrole-3-carboxylate $(\mathbf{4 g})$ Light Yellow; $58 \%$; m.p. $154-156^{\circ} \mathrm{C}$. IR (ATR) $v / \mathrm{cm}^{-1}: 3105(\mathrm{OH}), 2927(\mathrm{NH}$, amide), $1673.84(\mathrm{C}=\mathrm{O}$, ester), $1612(\mathrm{C}=\mathrm{C}), 1512(\mathrm{~N}-\mathrm{C}=\mathrm{O}), 776$ (Ar-OMe); ${ }^{1} \mathrm{H}-\mathrm{NMR}\left(400 \mathrm{MHz}, \mathrm{CDCl}_{3}\right): \delta 7.07(\mathrm{~d}, 2 \mathrm{H}), 6.86$ $(d, J=6.9 \mathrm{~Hz}, 2 \mathrm{H}), 4.95(\mathrm{~s}, 1 \mathrm{H}), 4.13(q, J=7.2 \mathrm{~Hz}$, $2 \mathrm{H}), 3.80(\mathrm{~s}, 3 \mathrm{H}), 2.79(\mathrm{~s}, 3 \mathrm{H}), 1.13(t, J=7.1 \mathrm{~Hz}$, $3 \mathrm{H}) ;{ }^{13} \mathrm{C}$ NMR $\left(100 \mathrm{MHz}, \mathrm{CDCl}_{3}\right): \delta 165.40(\mathrm{COH})$, $159.95(\mathrm{C}=\mathrm{O}), 157.87(\mathrm{C}=\mathrm{O}), 128.80(\mathrm{CH}-\mathrm{Ar})$, 126.47 (aromatic. C), 114.25 (CH-Ar), 112.12 (quat. C), $62.14\left(\mathrm{OCH}_{2}\right), 61.06(\mathrm{CH}), 55.39\left(\mathrm{OCH}_{3}\right), 27.65$ $\left(\mathrm{CH}_{3}-\mathrm{N}\right), 14.04\left(\mathrm{CH}_{3}\right) ;$ Anal. Calcd. for $\mathrm{C}_{15} \mathrm{H}_{17} \mathrm{NO}_{5} ; \mathrm{C}$, $61.85 ; \mathrm{H}, 5.88 ; \mathrm{N}, 4.81 ; \mathrm{O}, 27.46$. Found: $\mathrm{C}, 61.40 ; \mathrm{H}$, 5.81; N, 4.00; O, 28.69; GCMS $m / z$ (El, + ve): found $293.10\left([\mathrm{M}+2 \mathrm{H}]^{+}\right), \mathrm{C}_{15} \mathrm{H}_{17} \mathrm{NO}_{5}$ calculated 293.13.

Ethyl 4-hydroxy-2-(4-methoxyphenyl)-5-oxo-2,5dihydro-1H-pyrrole-3-carboxylate (4h)

Light yellow solid; $25.71 \%$; m.p. 152$153^{\circ} \mathrm{C}$. IR (ATR) $v / \mathrm{cm}^{-1}: 3302(\mathrm{OH}), 3004(\mathrm{NH}$, amide), 1685.55 ( $\mathrm{C}=\mathrm{O}$, ester), $1612(\mathrm{C}=\mathrm{C}), 1514.43$ ( $\mathrm{N}-\mathrm{C}=\mathrm{O}), 759(\mathrm{Ar}-\mathrm{OMe}) ;{ }^{1} \mathrm{H}-\mathrm{NMR}\left(400 \mathrm{MHz}, \mathrm{CDCl}_{3}\right)$ : $\delta 7.15(d d, J=6.6,2.1 \mathrm{~Hz}, 2 \mathrm{H}), 6.84(d d, J=6.6$, 
$2.1 \mathrm{~Hz}, 2 \mathrm{H}), 5.20(\mathrm{~d}, \mathrm{~J}=0.9 \mathrm{~Hz}, 1 \mathrm{H}), 4.15(q, J=$ $7.0 \mathrm{~Hz}, 2 \mathrm{H}), 3.78(\mathrm{~s}, 3 \mathrm{H}), 1.15(t, J=7.1 \mathrm{~Hz}, 3 \mathrm{H})$; ${ }^{13} \mathrm{C} \mathrm{NMR}\left(100 \mathrm{MHz}, \mathrm{CDCl}_{3}\right): \delta 170.70(\mathrm{COH}), 160.28$ $(\mathrm{C}=\mathrm{O}), 159.67(\mathrm{C}=\mathrm{O}), 137.20$ (aromatic. C) 135.93 (aromatic. C), 129.05 (CH-Ar), $114.32(\mathrm{CH}-\mathrm{Ar})$, 108.06 (quat. C), $64.17\left(\mathrm{OCH}_{2}\right), 55.39\left(\mathrm{OCH}_{3}\right), 39.98$ $(\mathrm{CH}), 13.89\left(\mathrm{CH}_{3}\right)$; Anal. Calcd. for $\mathrm{C}_{14} \mathrm{H}_{15} \mathrm{NO}_{5}$; C, 60.64; $\mathrm{H}, 5.45 ; \mathrm{N}, 5.05 ; \mathrm{O}, 28.85$. Found: $\mathrm{C}, 61.40 ; \mathrm{H}$, $5.81 ; \mathrm{N}, 4.00 ; \mathrm{O}, 28.79 ; \mathrm{GCMS} \mathrm{m} / \mathrm{z}(\mathrm{El},+\mathrm{ve})$ : found $278.10\left([\mathrm{M}+\mathrm{H}]^{+}\right), \mathrm{C}_{14} \mathrm{H}_{15} \mathrm{NO}_{5}$ calculated 278.10.

\section{Ethyl 4-hydroxy-1-(2-hydroxyethyl)-5-oxo-2,5- dihydro-1H-pyrrole-3-carboxylate (4i)}

Light orange; $34.60 \%$; m.p. $132-134^{\circ} \mathrm{C}$. IR (ATR) v/cm ${ }^{-1}: 3479\left(-\mathrm{CH}_{2} \mathrm{OH}\right), 3310(-\mathrm{CH}-\mathrm{OH})$, $2920(\mathrm{NH}$, amide $), 1694(\mathrm{C}=\mathrm{O}$, ester $), 1655(\mathrm{C}=\mathrm{C})$, 1513 (N-C=O); ${ }^{1} \mathrm{H}-\mathrm{NMR}\left(400 \mathrm{MHz}, \mathrm{CD}_{3} \mathrm{OD}\right): \delta 4.26$ (q, $J=7.2 \mathrm{~Hz}, 2 \mathrm{H}), 4.13(\mathrm{~s}, 2 \mathrm{H}), 3.76-3.68(\mathrm{~m}, 2 \mathrm{H})$, 3.62-3.51 (m, 2H), $1.29(t, J=7.1 \mathrm{~Hz}, 3 \mathrm{H}) ;{ }^{13} \mathrm{C}$ NMR (100 MHz, $\left.\mathrm{CD}_{3} \mathrm{OD}\right): \delta 176.98(\mathrm{COH}), 165.88(\mathrm{C}=\mathrm{O})$, $163.69(\mathrm{C}=\mathrm{O}), 107.90$ (quat. C), $60.32\left(\mathrm{OCH}_{2}\right), 59.42$ $\left(\mathrm{CH}_{2} \mathrm{OH}\right), 48.30\left(\mathrm{CH}_{2}-\mathrm{N}\right), 45.32\left(\mathrm{CH}_{2}\right), 13.27\left(\mathrm{CH}_{3}\right)$; Anal.Calcd. for $\mathrm{C}_{9} \mathrm{H}_{13} \mathrm{NO}_{5} ; \mathrm{C}, 50.23 ; \mathrm{H}, 6.09 ; \mathrm{N}, 6.51$; $\mathrm{O}$, 37.17. Found: C, 48.98; H, 5.91; N, 5.87; O, 39.24; GCMS $m / z(E l,+v e)$ : found $215.00\left([\mathrm{M}]^{+}\right), \mathrm{C}_{9} \mathrm{H}_{13} \mathrm{NO}_{5}$ calculated 215.08.

\section{Ethyl 1-butyl-4-hydroxy-5-oxo-2,5-dihydro-1H- pyrrole-3-carboxylate (4j)}

White Solid; $25 \%$; m.p. $109-111^{\circ} \mathrm{C}$. IR (ATR) $v / \mathrm{cm}^{-1}$ : $3099(\mathrm{OH}), 2956(\mathrm{NH}$, amide), $1663(\mathrm{C}=\mathrm{O}$, ester), $1447\left(-\mathrm{CH}_{2}-\right), 1362\left(-\mathrm{CH}_{3}\right) ;{ }^{1} \mathrm{H}-\mathrm{NMR}(400$ $\left.\mathrm{MHz}, \mathrm{CDCl}_{3}\right): \delta 4.30(q, J=7.2 \mathrm{~Hz}, 2 \mathrm{H}), 3.97(t, J=$ $15.8 \mathrm{~Hz}, 2 \mathrm{H}), 3.48(t, J=7.5 \mathrm{~Hz}, 2 \mathrm{H}), 1.57(\mathrm{~s}, 2 \mathrm{H})$, $1.32(t, J=7.3 \mathrm{~Hz}, 5 \mathrm{H}), 0.95-0.88(\mathrm{~m}, 3 \mathrm{H}) ;{ }^{13} \mathrm{C}$ NMR $\left(100 \mathrm{MHz}, \mathrm{CDCl}_{3}\right): \delta 165.09(\mathrm{COH}), 164.24(\mathrm{C}=\mathrm{O})$, $156.80(\mathrm{C}=\mathrm{O}), 107.68$ (quat. $\mathrm{C}), 61.15\left(\mathrm{OCH}_{2}\right), 46.24$ $\left(\mathrm{CH}_{2}-\mathrm{N}\right), 42.89\left(\mathrm{CH}_{2}\right), 30.35\left(\mathrm{CH}_{2}\right), 19.99\left(\mathrm{CH}_{2}\right)$, $14.35\left(\mathrm{CH}_{3}\right), 13.75\left(\mathrm{CH}_{3}\right)$; Anal. Calcd. for $\mathrm{C}_{11} \mathrm{H}_{17} \mathrm{NO}_{4}$; C, 58.14; H, 7.54; N, 6.16; O, 28.16. Found: C, 53.85; $\mathrm{H}, 7.04 ; \mathrm{N}, 5.06$; O, 34.05; GCMS m/z (El, + ve): found $227.10\left([\mathrm{M}]^{+}\right), \mathrm{C}_{11} \mathrm{H}_{17} \mathrm{NO}_{4}$ calculated 227.12.

Ethyl 4-hydroxy-1-(4-hydroxyphenyl)-5-oxo-2,5dihydro-1H-pyrrole-3-carboxylate (4k)

Brownish yellow solid; $5 \%$; m.p. $>160^{\circ} \mathrm{C}$ decomposed. IR (ATR) v/cm ${ }^{-1}: 3235(\mathrm{OH}), 2990$ ( $\mathrm{NH}$, amide), $1654(\mathrm{C}=\mathrm{O}$, ester), $1595(\mathrm{C}=\mathrm{C})$, 1514 (N-C=O), 757 (Ar-OH); ${ }^{1} \mathrm{H}-\mathrm{NMR}(400 \mathrm{MHz}$, $\left.\mathrm{CD}_{3} \mathrm{OD}\right): \delta 7.49(d, J=8.7 \mathrm{~Hz}, 2 \mathrm{H}), 6.80(d, J=9.1$ $\mathrm{Hz}, 2 \mathrm{H}), 4.40(\mathrm{~s}, 2 \mathrm{H}), 4.28(\mathrm{~d}, \mathrm{~J}=6.9 \mathrm{~Hz}, 2 \mathrm{H}), 1.31$ $(t, J=7.1 \mathrm{~Hz}, 3 \mathrm{H}) ;{ }^{13} \mathrm{C}$ NMR $\left(100 \mathrm{MHz}, \mathrm{CD}_{3} \mathrm{OD}\right): \delta$ $169.17(\mathrm{COH}), 166.68(\mathrm{C}=\mathrm{O}), 161.33(\mathrm{C}=\mathrm{O}), 155.31$ (quat. $\mathrm{C}$ ), 130.39 (quat. $\mathrm{C}$ ), 121.87 ( $\mathrm{CH}-\mathrm{Ar}$ ), 115.28 (CH-Ar), 110.34 (quat. C), $60.37\left(\mathrm{OCH}_{2}\right) 47.07\left(\mathrm{CH}_{2}\right)$, $13.32\left(\mathrm{CH}_{3}\right)$; Anal.Calcd. for $\mathrm{C}_{13} \mathrm{H}_{13} \mathrm{NO}_{5} ; \mathrm{C}, 59.31 ; \mathrm{H}$, 4.98; N, 5.32; O, 30.39. Found: C, 51.64; H, 4.92; N, 4.07; O, 29.37; GCMS $\mathrm{m} / \mathrm{z}(\mathrm{El},+\mathrm{ve})$ : found 286.90 $\left([\mathrm{M}+\mathrm{Na}]^{+}\right), \mathrm{C}_{13} \mathrm{H}_{13} \mathrm{NO}_{5}$ calculated 286.07.

\section{General procedure for the synthesis of pyrrolidine- based iminosugar intermediates (5a-c) \\ Mixtures of 0.01 mole of 2,3-dioxopyrrolidine} (4a), 0.012 mole of amine, 0.016 mole of formic acid were heated at reflux for 16 hours. Each solution was concentrated by distillation to approximately $10 \mathrm{~mL}$ and diluted with water while still hot until faint turbidity appeared. The products which crystallized from the mixture upon cooling, were collected by filtration and purified by recrystallization in ethanol-water mixture.

\section{Ethyl 5-oxo-4-(phenylamino)-2,5-dihydro-1H-} pyrrole-3-carboxylate (5a)

Yellow solid; $21.55 \%$; m.p. $59-62^{\circ} \mathrm{C}$. IR (ATR) $v / \mathrm{cm}^{-1}: 3396(\mathrm{NH}), 1700(\mathrm{C}=\mathrm{O}$, ester), 1675 $(\mathrm{C}=\mathrm{C}), 1539(\mathrm{~N}-\mathrm{C}=\mathrm{O}), 758(\mathrm{NH}-\mathrm{Ar}) ;{ }^{1} \mathrm{H}$ NMR $\left(400 \mathrm{MHz}, \mathrm{CDCl}_{3}\right): \delta 7.30(\mathrm{t}, 2 \mathrm{H}), 7.14(t, J=7.5$ $\mathrm{Hz}, 1 \mathrm{H}), 7.08(d, J=7.3 \mathrm{~Hz}, 2 \mathrm{H}), 4.93(\mathrm{~s}, 2 \mathrm{H}), 4.23$ $(q, J=7.2 \mathrm{~Hz}, 2 \mathrm{H}), 1.25(t, J=7.1 \mathrm{~Hz}, 3 \mathrm{H}) ;{ }^{13} \mathrm{C}$ NMR $\left(100 \mathrm{MHz}, \mathrm{CDCl}_{3}\right) 167.51(\mathrm{C}=\mathrm{O}), 163.89(\mathrm{C}=\mathrm{O})$, 137.85 (quat. C), 137.61 (aromatic. C), $128.72(\mathrm{CH}-$ Ar), 125.10 ( $\mathrm{CH}-\mathrm{Ar}), 122.73$ (CH-Ar), 111.83, (quat. C), $60.94\left(\mathrm{OCH}_{2}\right), 48.46\left(\mathrm{CH}_{2}\right), 14.28\left(\mathrm{CH}_{3}\right)$; GCMS $\mathrm{m} / \mathrm{z}(\mathrm{El},+\mathrm{ve})$ : found $247.10\left([\mathrm{M}+\mathrm{H}]^{+}\right), \mathrm{C}_{13} \mathrm{H}_{14} \mathrm{~N}_{2} \mathrm{O}_{3}$ calculated 247.11.

\section{Ethyl 4-((4-ethylphenyl)amino)-5-oxo-2,5-dihydro- 1H-pyrrole-3-carboxylate (5b)}

Reddish brown oily; 36.06\%; IR (ATR) $v / \mathrm{cm}^{-1}: 3328(\mathrm{NH}), 1766(\mathrm{C}=\mathrm{O}$, ester), $1685(\mathrm{C}=\mathrm{C})$, $1517(\mathrm{~N}-\mathrm{C}=\mathrm{O}), 1459\left(\mathrm{CH}_{2}\right), 1356\left(\mathrm{CH}_{3}\right), 759$ $\left(\mathrm{NH}-\mathrm{Ar}-\mathrm{CH}_{2} \mathrm{CH}_{3}\right) ;{ }^{1} \mathrm{H}-\mathrm{NMR}\left(400 \mathrm{MHz}, \mathrm{CDCl}_{3}\right): \delta$ $7.13(d, J=8.5 \mathrm{~Hz}, 2 \mathrm{H}), 7.01(d, J=6.2 \mathrm{~Hz}, 2 \mathrm{H})$, $4.93(\mathrm{~s}, 2 \mathrm{H}), 4.23(q, J=7.2 \mathrm{~Hz}, 2 \mathrm{H}), 2.62(q, J=$ $7.6 \mathrm{~Hz}, 2 \mathrm{H}), 1.28-1.19(\mathrm{~m}, 6 \mathrm{H}) ;{ }^{13} \mathrm{C}$ NMR $(100 \mathrm{MHz}$, $\left.\mathrm{CDCl}_{3}\right) \delta 141.35(\mathrm{C}=\mathrm{O}), 137.92(\mathrm{C}=\mathrm{O}), 135.36$ (quat. C), 128.12 (CH-Ar), 122.98 (CH-Ar), 119.530 (aromatic. C), 113.79 (aromatic. C), 110.92 (quat. C), $60.82\left(\mathrm{OCH}_{2}\right), 28.39\left(\mathrm{CH}_{2}\right), 15.59\left(\mathrm{CH}_{3}\right), 14.29$ $\left(\mathrm{CH}_{3}\right)$; GCMS $\mathrm{m} / \mathrm{z}(\mathrm{El},+\mathrm{ve})$ : found $275.10\left([\mathrm{M}+\mathrm{H}]^{+}\right)$, $\mathrm{C}_{15} \mathrm{H}_{18} \mathrm{~N}_{2} \mathrm{O}_{3}$ calculated 275.14. 
Ethyl 4-((4-methoxyphenyl) amino)-5-oxo-2,5dihydro-1 H-pyrrole-3-carboxylate (5c)

Light yellow solid; $25.05 \%$; m.p. $70-71^{\circ} \mathrm{C}$. IR (ATR) v/cm-1: $3327(\mathrm{NH}), 2982(\mathrm{NH}$, amide), 1766 (C=O, ester), $1638(\mathrm{C}=\mathrm{C}), 1517$ ( $\mathrm{N}-\mathrm{C}=\mathrm{O}), 755$ ( $\mathrm{NH}-\mathrm{Ar}-\mathrm{OMe}) ;{ }^{1} \mathrm{H} \mathrm{NMR}\left(400 \mathrm{MHz}, \mathrm{CD}_{3} \mathrm{COCD}_{3}\right) 8.79$ $(d, J=10.9 \mathrm{~Hz}, 2 \mathrm{H}), 8.54(d, J=11.2 \mathrm{~Hz}, 2 \mathrm{H}), 6.14$ (s, 2H), 5.30 (q, J = $8.9 \mathrm{~Hz}, 2 \mathrm{H}), 4.73(\mathrm{~s}, 3 \mathrm{H}), 1.59$ $(t, J=8.9 \mathrm{~Hz}, 3 \mathrm{H}) ;{ }^{13} \mathrm{C} \mathrm{NMR}\left(100 \mathrm{MHz}, \mathrm{CD}_{3} \mathrm{COCD}_{3}\right) \delta$ $178.90(\mathrm{C}=\mathrm{O}), 166.36(\mathrm{C}=\mathrm{O}), 159.38$ (aromatic. $\mathrm{C}$ ), 157.52 (quat. C), 143.66 (aromatic. C), 130.63 (quat. C), 125.11 (CH-Ar), 113.93 (quat. C), $67.56\left(\mathrm{OCH}_{2}\right)$, $60.79\left(\mathrm{CH}_{2}\right), 55.54\left(\mathrm{OCH}_{3}\right), 14.35\left(\mathrm{CH}_{3}\right) ; \mathrm{GCMS}$ $\mathrm{m} / \mathrm{z}(\mathrm{El},+\mathrm{ve})$ : found $277.10\left([\mathrm{M}+\mathrm{H}]^{+}\right), \mathrm{C}_{14} \mathrm{H}_{16} \mathrm{~N}_{2} \mathrm{O}_{4}$ calculated 277.12.

\section{General procedure for the synthesis of pyrrolidine- based iminosugar intermediates (6a-e)}

Mixtures consisting 1 eq 6a-e were stirred in ethanol with $\mathrm{Pd}-\mathrm{C}$ ( $10 \%$ wt.) ( 0.5 equiv.) and acetic acid (2 equiv.). The reactions were stirred vigorously under hydrogen atmosphere towards completion (24 h) and then filtered through Celite. After removal of the solvent, the crude products were used in following steps without further purification

\section{Ethyl 4-hydroxy-5-oxopyrrolidine-3-carboxylate (6a)}

Colourless oily; $58 \%$; IR (ATR) $v / \mathrm{cm}^{-1}: 3392$ (OH), 2915 ( $\mathrm{NH}$, amide), 1779 ( $\mathrm{C}=\mathrm{O}$, ester), 1470 $\left(\mathrm{CH}_{2}\right), 1352\left(\mathrm{CH}_{3}\right) ;{ }^{1} \mathrm{H}$ NMR $\left(400 \mathrm{MHz}, \mathrm{CDCl}_{3}\right) \delta$ $4.62(d, J=7.8 \mathrm{~Hz}, 1 \mathrm{H}), 4.55(d, J=12.0 \mathrm{~Hz}, 1 \mathrm{H})$, $4.44-4.25(\mathrm{~m}, 1 \mathrm{H}), 4.23(t, J=10.7 \mathrm{~Hz}, 2 \mathrm{H}), 3.65$ $-3.25(\mathrm{~m}, 1 \mathrm{H}), 1.28\left(t, J=7.1 \mathrm{~Hz}, 3 \mathrm{H} ;{ }^{13} \mathrm{C}\right.$ NMR $\left(100 \mathrm{MHz}, \mathrm{CDCl}_{3}\right) \delta 154.58(\mathrm{C}=\mathrm{O}), 145.25(\mathrm{C}=\mathrm{O})$, $68.38(\mathrm{CHOH}), 66.39\left(\mathrm{OCH}_{2}\right), 62.10\left(\mathrm{CH}_{2}\right), 45.61$ (CH), $14.17\left(\mathrm{CH}_{3}\right)$; GCMS m/z (El, + ve): found $174.00\left([\mathrm{M}+\mathrm{H}]^{+}\right), \mathrm{C}_{7} \mathrm{H}_{11} \mathrm{NO}_{4}$ calculated 174.08.

\section{Ethyl 4-hydroxy-1-methyl-5-oxopyrrolidine-3- carboxylate (6b)}

White solid; $98 \%$; m.p. $116-118^{\circ} \mathrm{C}$. IR (ATR) $v / \mathrm{cm}^{-1}: 3217(\mathrm{OH}), 2989(\mathrm{NH}$, amide), $1721.73(\mathrm{C}=\mathrm{O}$, ester), $1503(\mathrm{~N}-\mathrm{C}=\mathrm{O}), 1463\left(\mathrm{CH}_{2}\right), 1353\left(\mathrm{CH}_{3}\right)$; ${ }^{1} \mathrm{H}$ NMR (400 MHz, $\left.\mathrm{CD}_{3} \mathrm{OD}\right): \delta 4.44(d, J=7.8 \mathrm{~Hz}$, $1 \mathrm{H}$ ), 4.15 (q, $J=7.2 \mathrm{~Hz}, 2 \mathrm{H}$ ), $3.64(d d, J=10.1$, $3.2 \mathrm{~Hz}, 1 \mathrm{H}), 3.28(t, J=1.6 \mathrm{~Hz}, 1 \mathrm{H}), 3.05(\mathrm{~s}, 1 \mathrm{H})$, $2.84(d, J=5.5 \mathrm{~Hz}, 3 \mathrm{H}), 1.28-1.20(\mathrm{~m}, 3 \mathrm{H}) ;{ }^{13} \mathrm{C}$ NMR (100 MHz, CD $\left.{ }_{3} \mathrm{OD}\right): \delta 172.93(\mathrm{C}=\mathrm{O}), 170.711$ $(\mathrm{C}=\mathrm{O}), 70.27(\mathrm{CHOH}), 60.71\left(\mathrm{OCH}_{2}\right), 47.88\left(\mathrm{CH}_{2}\right)$, $43.46(\mathrm{CH}), 28.70\left(\mathrm{CH}_{3}-\mathrm{N}\right), 13.15\left(\mathrm{CH}_{3}\right)$; Anal. Calcd. for $\mathrm{C}_{8} \mathrm{H}_{13} \mathrm{NO}_{4}$ : $\mathrm{C}, 51.33 ; \mathrm{H}, 7.00 ; \mathrm{N}, 7.48 ; \mathrm{O}$,
34.19. Found: C, 43.95; H, 6.13; N, 5.86; GCMS m/z (El, + ve): found $187.10\left([\mathrm{M}]^{+}\right), \mathrm{C}_{8} \mathrm{H}_{13} \mathrm{NO}_{4}$ calculated 187.08 .

\section{Ethyl 5-oxo-4-(phenylamino)pyrrolidine-3- carboxylate (6c)}

Light yellow solid; $31.80 \%$; m.p. $97-99^{\circ} \mathrm{C}$. IR (ATR) v/cm ${ }^{-1}: 3345.19(\mathrm{NH}), 3007(\mathrm{NH}$, amide), 2985 ( $\mathrm{CH}$, aromatic) $1769.48(\mathrm{C}=\mathrm{O}$, ester), $1514(\mathrm{~N}-\mathrm{C}=\mathrm{O})$, $1470\left(\mathrm{CH}_{2}\right), 1305\left(\mathrm{CH}_{3}\right), 751(\mathrm{NH}-\mathrm{Ar}) ;{ }^{1} \mathrm{H}-\mathrm{NMR}(400$ $\mathrm{MHz}, \mathrm{CDCl}_{3}$ ): $\delta 7.21$ (qd, 2H), 6.80 (qt, 1H), 6.67 $(d, J=7.3 \mathrm{~Hz}, 2 \mathrm{H}), 4.59$ (dd, $J=9.6,0.9 \mathrm{~Hz}, 1 \mathrm{H})$, 4.45-4.36 (m, 2H), 4.14-4.00 (m, 2H), 3.81-3.73 $(\mathrm{m}, 1 \mathrm{H}), 1.05(t, J=7.3 \mathrm{~Hz}, 3 \mathrm{H}) ;{ }^{13} \mathrm{C}$ NMR $(100$ $\left.\mathrm{MHz}, \mathrm{CDCl}_{3}\right) \delta 174.19(\mathrm{C}=\mathrm{O}), 170.24(\mathrm{C}=\mathrm{O}), 146.25$ (aromatic. C), 129.43 (CH-Ar), 119.40 (quat. C), 113.70 (CH-Ar), $66.99\left(\mathrm{OCH}_{2}\right), 61.62\left(\mathrm{CH}_{2}\right), 55.41(\mathrm{CH}), 46.17$ (CH), $13.95\left(\mathrm{CH}_{3}\right)$; GCMS m/z (El, + ve): found 249.10 $\left([\mathrm{M}+\mathrm{H}]^{+}\right), \mathrm{C}_{13} \mathrm{H}_{16} \mathrm{~N}_{2} \mathrm{O}_{3}$ calculated 249.12.

\section{Ethyl 4-((4-ethylphenyl)amino)-5-oxopyrrolidine- 3-carboxylate (6d)}

Reddish yellow solid; $23.54 \%$; m.p. 81-83 ${ }^{\circ} \mathrm{C}$. IR (ATR) v/cm ${ }^{-1}: 3345.10(\mathrm{NH}), 3007.85$ ( $\mathrm{NH}$, amide), $2980(\mathrm{CH}$, aromatic), 1770.48 (ester), $1514(\mathrm{~N}-\mathrm{C}=\mathrm{O}), 1452\left(\mathrm{CH}_{2}\right), 1376\left(\mathrm{CH}_{3}\right), 690(\mathrm{NH}-$ Ar); ${ }^{1} \mathrm{H}-\mathrm{NMR}\left(400 \mathrm{MHz}, \mathrm{CDCl}_{3}\right): 7.04(d, J=8.7$ $\mathrm{Hz}, 2 \mathrm{H}), 6.62(d, J=8.2 \mathrm{~Hz}, 2 \mathrm{H}), 4.60(d, J=10.1$ $\mathrm{Hz}, 1 \mathrm{H}), 4.42(d d, J=10.1,5.9 \mathrm{~Hz}, 1 \mathrm{H}), 4.34(q, J$ $=4.1 \mathrm{~Hz}, 1 \mathrm{H}), 4.10(q d, J=7.1,2.6 \mathrm{~Hz}, 2 \mathrm{H}), 3.78-$ $3.72(\mathrm{~m}, 1 \mathrm{H}), 2.54(q, J=7.6 \mathrm{~Hz}, 2 \mathrm{H}), 1.18(q, J$ $=7.3 \mathrm{~Hz}, 3 \mathrm{H}$ ), 1.08 (t, J=7.3 Hz, 3H); ${ }^{13} \mathrm{C}$ NMR $\left(100 \mathrm{MHz}, \mathrm{CDCl}_{3}\right) \delta 174.20(\mathrm{C}=\mathrm{O}), 170.17(\mathrm{C}=\mathrm{O})$, 144.16 (aromatic. C), 135.38 (aromatic. C), 128.75 (CH-Ar), $113.87(\mathrm{CH}-\mathrm{Ar}), 66.93\left(\mathrm{OCH}_{2}\right), 61.62\left(\mathrm{CH}_{2}\right)$, $55.85(\mathrm{CH}), 46.37(\mathrm{CH}), 28.05\left(\mathrm{CH}_{2}\right), 16.01\left(\mathrm{CH}_{3}\right)$, $14.00\left(\mathrm{CH}_{3}\right) ; \mathrm{GCMS} \mathrm{m} / \mathrm{z}(\mathrm{El},+\mathrm{ve})$ : found 277.10 $\left([\mathrm{M}+\mathrm{H}]^{+}\right), \mathrm{C}_{15} \mathrm{H}_{20} \mathrm{~N}_{2} \mathrm{O}_{3}$ calculated 277.15.

\section{Ethyl 4-((4-methoxyphenyl)amino)-5-oxopyrrolidine- 3-carboxylate (6e)}

Yellow solid; $32.5 \%$; m.p. $102-104^{\circ} \mathrm{C}$. IR (ATR) v/cm-1: $3345(\mathrm{NH}), 3007$ (NH, amide), 2980 ( $\mathrm{CH}$, aromatic), $1769(\mathrm{C}=\mathrm{O})$, ester), $1514(\mathrm{~N}-\mathrm{C}=\mathrm{O})$, $1460\left(\mathrm{CH}_{2}\right), 1376\left(\mathrm{CH}_{3}\right), 819(\mathrm{NH}-\mathrm{Ar}) ;{ }^{1} \mathrm{H}-\mathrm{NMR}$ $\left(400 \mathrm{MHz}, \mathrm{CDCl}_{3}\right): \delta 6.79(d d, J=6.9,2.3 \mathrm{~Hz}, 2 \mathrm{H})$, 6.68-6.63 (m, 2H), $4.60(d, J=10.1 \mathrm{~Hz}, 1 \mathrm{H}), 4.41$ $(d d, J=10.1,5.9 \mathrm{~Hz}, 1 \mathrm{H}), 4.30(q, J=4.1 \mathrm{~Hz}, 1 \mathrm{H})$, 4.15-4.04 (m, 3H), $3.74(d, J=3.7 \mathrm{~Hz}, 3 \mathrm{H}), 1.09$ $(t, J=7.1 \mathrm{~Hz}, 3 \mathrm{H}) ;{ }^{13} \mathrm{C} \mathrm{NMR}\left(100 \mathrm{MHz}, \mathrm{CDCl}_{3}\right): \delta$ $174.30(\mathrm{C}=\mathrm{O}), 172.22(\mathrm{C}=\mathrm{O}), 153.44$ (aromatic. $\mathrm{C})$, 
140.32 (aromatic. C), 115.27 (CH-Ar), $114.13(\mathrm{CH}-$ Ar), $66.91\left(\mathrm{OCH}_{2}\right), 61.62\left(\mathrm{CH}_{2}\right), 56.46(\mathrm{CH}), 55.79$ $\left(\mathrm{OCH}_{3}\right), 46.38(\mathrm{CH}), 14.02\left(\mathrm{CH}_{3}\right) ; \mathrm{GCMS} \mathrm{m} / \mathrm{z}(\mathrm{El},+$ ve): found $279.10\left([\mathrm{M}+\mathrm{H}]^{+}\right), \mathrm{C}_{14} \mathrm{H}_{18} \mathrm{~N}_{2} \mathrm{O}_{4}$ calculated 279.13.

General procedure for the synthesis of pyrrolidinebased iminosugars intermediates (8a-e)

A stirred mixture of $4 \mathrm{c}-\mathrm{g}$ (1 eq.) in $\mathrm{CH}_{2} \mathrm{Cl}_{2}$ $(0.05 \mathrm{~L})$ was added acetic acid (1 eq.) and $\mathrm{NaBH}_{4}$ $(1.1 \mathrm{~mol})$ at $0^{\circ} \mathrm{C}$. After $0^{\circ} \mathrm{C}$ achieved, the mixture was stirred for one hour, then the mixture was stirred at room temperature upon completion (8 hours). The solvent was then removed from mixtures and was extracted with EtOAc. The organic layer was washed with highly saturated $\mathrm{NaHCO}_{3}$ solution. Trace of water was removed with anhydrous $\mathrm{MgSO}_{4}$ and concentrated in vacuo. The crude product was purified by column chromatography to give the hydroxy ester product (8a-e).

\section{Ethyl-2-ethyl-4-hydroxy-5-oxopyrrolidine-3- carboxylate(8a)}

White solid; 99\%; m.p. $112-114^{\circ} \mathrm{C}$. IR (ATR)

$v / \mathrm{cm}^{-1}$ : $3312(\mathrm{OH}), 3184(\mathrm{NH}$, amide), $1709(\mathrm{C}=\mathrm{O}$, ester) $1459\left(\mathrm{CH}_{2}\right), 1371\left(\mathrm{CH}_{3}\right) ;{ }^{1} \mathrm{H}-\mathrm{NMR}(400 \mathrm{MHz}$, $\left.\mathrm{CD}_{3} \mathrm{OD}\right): \delta 4.47(t, J=9.8 \mathrm{~Hz}, 1 \mathrm{H}), 4.22(\mathrm{~s}, 2 \mathrm{H})$, 3.60 (s, 1H), $2.71(d, J=11.0 \mathrm{~Hz}, 1 \mathrm{H}), 1.74-1.58$ $(\mathrm{m}, 2 \mathrm{H}), 1.35-1.23(\mathrm{~m}, 3 \mathrm{H}), 0.95(d d, J=18.5,7.5 \mathrm{~Hz}$, $3 \mathrm{H}) ;{ }^{13} \mathrm{C}$ NMR (100 MHz, CD $\left.\mathrm{OD}\right) \delta 175.40$ (C=O), $172.19(\mathrm{C}=\mathrm{O}), 72.74(\mathrm{CHOH}), 61.12\left(\mathrm{OCH}_{2}\right), 54.76$ $(\mathrm{CH}), 54.42(\mathrm{CH}), 27.89\left(\mathrm{CH}_{2}\right), 13.17\left(\mathrm{CH}_{3}\right), 8.22$ $\left(\mathrm{CH}_{3}\right)$; LCMS $\mathrm{m} / \mathrm{z}(\mathrm{H}-\mathrm{ESI},+\mathrm{ve})$ : found 202.1072 $\left([\mathrm{M}+\mathrm{H}]^{+}\right), \mathrm{C}_{9} \mathrm{H}_{15} \mathrm{NO}_{4}$ calculated 202.1073.

\section{Ethyl-4-hydroxy-2-isopropyl-5-oxopyrrolidine-3- carboxylate(8b)}

White solid; $99 \%$; m.p. $>175^{\circ} \mathrm{C}$ decomposed. IR (ATR) $v / \mathrm{cm}^{-1}: 3366(\mathrm{OH}), 2971$ (NH, amide), 1726 (C=O, ester), $1682(\mathrm{~N}-\mathrm{C}=\mathrm{O}), 1466\left(\mathrm{CH}_{2}\right), 1375\left(\mathrm{CH}_{3}\right)$; ${ }^{1} \mathrm{H}-\mathrm{NMR}\left(400 \mathrm{MHz}, \mathrm{CD}_{3} \mathrm{OD}\right) \delta$ 4.44-4.37 (1H), 4.23$4.11(2 \mathrm{H}), 3.55-3.48(1 \mathrm{H}), 3.34-3.31(1 \mathrm{H}), 2.80-2.71$ $(1 \mathrm{H}), 1.85-1.74(1 \mathrm{H}), 1.30-1.22(3 \mathrm{H}), 0.98-0.82$ $(6 \mathrm{H}) ;{ }^{13} \mathrm{C}$ NMR $\left(100 \mathrm{MHz}, \mathrm{CD}_{3} \mathrm{OD}\right): \delta 175.28(\mathrm{C}=\mathrm{O})$, $172.80(\mathrm{C}=\mathrm{O}), 73.23(\mathrm{CHOH}), 61.12\left(\mathrm{OCH}_{2}\right), 58.83$ $(\mathrm{CH}), 52.39(\mathrm{CH}), 31.81(\mathrm{CH}), 16.77\left(\mathrm{CH}_{3} \times 2\right), 13.09$ $\left(\mathrm{CH}_{3}\right)$; LCMS $\mathrm{m} / \mathrm{z}(\mathrm{H}-\mathrm{ESI},+\mathrm{ve})$ : found 216.1228 $\left([\mathrm{M}+\mathrm{H}]^{+}\right), \mathrm{C}_{10} \mathrm{H}_{17} \mathrm{NO}_{4}$ calculated 216.1230.

Ethyl-4-hydroxy-5-oxo-2-(p-tolyl)pyrrolidine-3carboxylate(8c)

White solid; $94.44 \%$; m.p. $189-200^{\circ} \mathrm{C}$. IR
(ATR) v/cm-1: $3294(\mathrm{OH}), 3054$ (NH, amide), 2981 ( $\mathrm{CH}$, aromatic), $1729(\mathrm{C}=\mathrm{O}$, ester), $1518(\mathrm{~N}-\mathrm{C}=\mathrm{O})$, $1458\left(\mathrm{CH}_{2}\right), 642\left(\mathrm{Ar}-\mathrm{CH}_{3}\right) ;{ }^{1} \mathrm{H}-\mathrm{NMR}(400 \mathrm{MHz}$, $\left.\mathrm{CD}_{3} \mathrm{OD}\right): \delta$ 7.25-7.15 $(4 \mathrm{H}), 4.66-4.63(1 \mathrm{H}), 4.58-$ $4.53(1 \mathrm{H}), 4.22-4.04(2 \mathrm{H}), 2.94-2.84(1 \mathrm{H}), 2.35-2.26$ (3H), 1.21-1.14 (3H); ${ }^{13} \mathrm{C}$ NMR (100 MHz, CD 3 OD): \& $175.47(\mathrm{C}=\mathrm{O}), 171.42(\mathrm{C}=\mathrm{O}), 138.24$ (aromatic. C), 136.98 (aromatic. C), 127.68 ( $\mathrm{CH}-\mathrm{Ar}), 126.19$ (CH-Ar), $72.69(\mathrm{CHOH}), 61.08(\mathrm{CH}), 59.08(\mathrm{CH}), 56.53$ (CH), $19.82\left(\mathrm{CH}_{3}\right), 13.12\left(\mathrm{CH}_{3}\right)$; GCMS $\mathrm{m} / \mathrm{z}(\mathrm{El},+\mathrm{ve})$ : found $263.10\left([\mathrm{M}]^{+}\right), \mathrm{C}_{14} \mathrm{H}_{17} \mathrm{NO}_{4}$ calculated 263.12.

\section{Ethyl-2-(4-cyanophenyl)-4-hydroxy-5-oxopyrrolidine- 3-carboxylate(8d)}

White solid; $82.73 \%$; m.p. $>231^{\circ} \mathrm{C}$ decomposed. IR (ATR) $v / \mathrm{cm}^{-1}: 3200(\mathrm{OH}), 1675$ (C=O, ester), $1476\left(\mathrm{CH}_{2}\right), 637$ (Ar-CN); ${ }^{1} \mathrm{H}-\mathrm{NMR}$ (400 MHz, CD $\left.{ }_{3} \mathrm{OD}\right): \delta 7.75(d, J=8.2 \mathrm{~Hz}, 2 \mathrm{H})$, $7.54(d, J=8.2 \mathrm{~Hz}, 2 \mathrm{H}), 4.80(d, J=8.2 \mathrm{~Hz}, 1 \mathrm{H})$, $4.58(d, J=9.1 \mathrm{~Hz}, 1 \mathrm{H}), 4.17(t, J=7.3 \mathrm{~Hz}, 2 \mathrm{H})$, $2.88(t, J=8.7 \mathrm{~Hz}, 1 \mathrm{H}), 1.20(t, J=7.1 \mathrm{~Hz}, 3 \mathrm{H})$; ${ }^{13} \mathrm{C}$ NMR $\left(100 \mathrm{MHz}, \mathrm{CD}_{3} \mathrm{OD}\right): \delta 175.54(\mathrm{C}=\mathrm{O}), 175.10$ (C=O), 152.07 (aromatic. C), 132.48 (CH-Ar), 129.96 (CH-Ar), $127.45(\mathrm{CN}), 110.78$ (aromatic. C), 73.92 $(\mathrm{CHOH}), 58.84\left(\mathrm{OCH}_{2}\right), 43.97(\mathrm{CH}), 42.24(\mathrm{CH})$, $11.85\left(\mathrm{CH}_{3}\right) ; \mathrm{GCMS} \mathrm{m} / \mathrm{z}(\mathrm{EI},+\mathrm{ve})$ : found 297.00 $\left([\mathrm{M}+\mathrm{Na}]^{+}\right), \mathrm{C}_{14} \mathrm{H}_{14} \mathrm{~N}_{2} \mathrm{O}_{4}$ calculated 297.08.

\section{Ethyl-4-hydroxy-2-(4-methoxyphenyl)-1-methyl- 5-oxopyrrolidine-3-carboxylate(8e)}

Yellow solid; $99 \%$; m.p. $54-55^{\circ} \mathrm{C}$. IR (ATR) $v / \mathrm{cm}^{-1}: 3217(\mathrm{OH}), 2989(\mathrm{CH}$, aromatic), $1721(\mathrm{C}=\mathrm{O}$, ester), $1503(\mathrm{~N}-\mathrm{C}=\mathrm{O}), 1473\left(\mathrm{CH}_{2}\right), 688(\mathrm{Ar}-\mathrm{OMe})$; ${ }^{1} \mathrm{H}-\mathrm{NMR}\left(400 \mathrm{MHz}, \mathrm{CD}_{3} \mathrm{OD}\right): \delta 7.19(d, J=8.7 \mathrm{~Hz}$, $2 \mathrm{H}), 6.89(d, J=8.7 \mathrm{~Hz}, 2 \mathrm{H}), 4.80-4.75(\mathrm{~m}, 1 \mathrm{H})$, $4.55(d, J=7.3 \mathrm{~Hz}, 1 \mathrm{H}), 3.84-3.70(\mathrm{~m}, 5 \mathrm{H}), 3.62$ $(t, J=7.1 \mathrm{~Hz}, 1 \mathrm{H}), 2.69(d, J=7.3 \mathrm{~Hz}, 3 \mathrm{H}), 0.90$ $(t, J=7.1 \mathrm{~Hz}, 3 \mathrm{H}) ;{ }^{13} \mathrm{C}$ NMR $\left(100 \mathrm{MHz}, \mathrm{CD}_{3} \mathrm{OD}\right) \delta$ $169.07(\mathrm{C}=\mathrm{O}), 168.04(\mathrm{C}=\mathrm{O}), 160.06$ (aromatic. $\mathrm{C}$ ), 129.51 (CH-Ar), 126.86 (aromatic. C), $113.55(\mathrm{CH}-$ $\mathrm{Ar}), 70.30(\mathrm{CHOH}), 61.83(\mathrm{CH}), 60.22\left(\mathrm{OCH}_{2}\right), 54.42$ $\left(\mathrm{OCH}_{3}\right), 51.32(\mathrm{CH}), 27.92\left(\mathrm{CH}_{3}-\mathrm{N}\right), 12.79\left(\mathrm{CH}_{3}\right)$; Anal. Calcd. for $\mathrm{C}_{14} \mathrm{H}_{17} \mathrm{NO}_{5}$ : C, 60.21; $\mathrm{H}, 6.14 ; \mathrm{N}, 5.02$; O, 28.64. Found: C, 57.68; H, 2.84; N, 6.59; GCMS $\mathrm{m} / \mathrm{z}(\mathrm{El},+\mathrm{ve})$ : found $295.10\left([\mathrm{M}+2 \mathrm{H}]^{+}\right), \mathrm{C}_{15} \mathrm{H}_{19} \mathrm{NO}_{5}$ calculated 295.14.

\section{General procedure for the synthesis of pyrrolidine- based iminosugars (7a-e) and (9a-e) \\ The crude products obtained were dissolved in dry tetrahydrofuran and were added}


slowly to the lithium aluminium hydride solution (excess) in inert atmosphere. The mixtures were heated at $90^{\circ} \mathrm{C}$ towards completion (4-8 h) and then cooled to $0^{\circ} \mathrm{C}$. The reaction mixtures were quenched by adding of distilled water, and the mixtures were filtered through Celite and concentrated in vacuo to give the crude products iminosugar derivatives. Compounds (7a-e) and (9a-e) as oil after purification by column chromatography.

\section{4-(hydroxymethyl)pyrrolidin-3-ol (7a)}

Reddish oily; 81.23\%; IR (ATR) v/cm ${ }^{-1}: 3278$ (CH-OH), 2927 (NH, stretch), $1654(\mathrm{NH}$, bend), 1412 $\left(\mathrm{CH}_{2}\right), 1016$ (C-O); ${ }^{1} \mathrm{H}-\mathrm{NMR}$ (400 MHz, CD $\left.\mathrm{OD}\right): \delta$ 3.81-3.72 (m, 2H), $3.67(q, J=5.6 \mathrm{~Hz}, 3 \mathrm{H}), 3.58$ (td, $J=11.5,5.5 \mathrm{~Hz}, 2 \mathrm{H}), 1.78(q, J=5.6 \mathrm{~Hz}, 1 \mathrm{H})$; ${ }^{13} \mathrm{C} \mathrm{NMR}\left(100 \mathrm{MHz}, \mathrm{CD}_{3} \mathrm{OD}\right): \delta 71.30(\mathrm{CHOH}), 64.32$ $\left(\mathrm{CH}_{2}\right), 60.28\left(\mathrm{CH}_{2} \mathrm{OH}\right), 59.69(\mathrm{CH}), 45.75(\mathrm{CH})$; LCMS m/z (ESI-QTOF, + ve): found 119.0794 ([M + $\left.2 \mathrm{H}]^{+}\right), \mathrm{C}_{5} \mathrm{H}_{11} \mathrm{NO}_{2}$ calculated 119.0941 .

\section{4-(hydroxymethyl)-1-methylpyrrolidin-3-ol (7b):}

Reddish oily; 64.27\%; IR (ATR) v/cm ${ }^{-1}: 3254$

$(\mathrm{OH}), 2948$ (NH, stretch), 1654 (NH, bend), 1407 $\left(\mathrm{CH}_{2}\right), 1021$ (C-O); ${ }^{1} \mathrm{H}$ NMR (400 MHz, CD $\left.\mathrm{OD}\right): \delta$ $4.36(t d, J=6.1,3.8 \mathrm{~Hz}, 1 \mathrm{H}), 3.77$ (dd, $J=11.0,6.4$ $\mathrm{Hz}, 1 \mathrm{H}), 3.60(d d, J=11.0,7.3 \mathrm{~Hz}, 1 \mathrm{H}), 3.05(q, J=$ $5.5 \mathrm{~Hz}, 1 \mathrm{H}), 2.84(d d, J=9.6,7.8 \mathrm{~Hz}, 1 \mathrm{H}), 2.53-2.43$ $(\mathrm{m}, 2 \mathrm{H}), 2.38(\mathrm{~s}, 3 \mathrm{H}), 1.90-1.80(\mathrm{~m}, 1 \mathrm{H}) ;{ }^{13} \mathrm{C} \mathrm{NMR}$ (100 MHz, CD $\mathrm{OD}): \delta 71.17(\mathrm{CHOH}), 63.87\left(\mathrm{CH}_{2}\right)$, $60.17\left(\mathrm{CH}_{2} \mathrm{OH}\right), 57.42\left(\mathrm{CH}_{2}\right), 45.31\left(\mathrm{CH}_{3}-\mathrm{N}\right), 41.56$ (CH); LCMS m/z (ESI-QTOF, + ve): found 132.1044 $\left([\mathrm{M}+\mathrm{H}]^{+}\right), \mathrm{C}_{6} \mathrm{H}_{13} \mathrm{NO}_{2}$ calculated 132.1019.

\section{(4-(phenylamino)pyrrolidin-3-ol) (7c)}

Yellow solid; 22.19\%; IR (ATR) v/cm ${ }^{-1}: 3345$ $(\mathrm{OH}), 2917$ ( $\mathrm{NH}$, stretch), 2830 ( $\mathrm{CH}$, aromatic), 1599 (NH, bend), $1496\left(\mathrm{CH}_{2}\right), 1020$ (C-O), 692 (NH-Ar); ${ }^{1} \mathrm{H}-\mathrm{NMR}\left(400 \mathrm{MHz}, \mathrm{CD}_{3} \mathrm{OD}\right): \delta 7.05$ (dd, $J=8.7,7.3$ $\mathrm{Hz}, 2 \mathrm{H}), 6.64(d d, J=7.8 \mathrm{~Hz}, 2 \mathrm{H}), 6.55(t t, J=7.1 \mathrm{~Hz}$, $1 \mathrm{H}), 3.75(d d, J=5.5,3.7 \mathrm{~Hz}, 2 \mathrm{H}), 3.70(d, J=5.9 \mathrm{~Hz}$, $2 \mathrm{H}), 3.66(\mathrm{~s}, 3 \mathrm{H}), 2.02-1.93(\mathrm{~m}, 1 \mathrm{H}) ;{ }^{13} \mathrm{C}$ NMR (100 $\left.\mathrm{MHz}, \mathrm{CD}_{3} \mathrm{OD}\right): \delta 148.39$ (aromatic. $\left.\mathrm{C}\right), 128.76(\mathrm{CH}-$ Ar), 116.50 (CH-Ar), $113.04(\mathrm{CH}-\mathrm{Ar}), 61.37\left(\mathrm{CH}_{2} \mathrm{OH}\right)$, $60.05\left(\mathrm{CH}_{2}\right), 53.76(\mathrm{CHNH}), 44.67(\mathrm{CH}), 29.43\left(\mathrm{CH}_{2}\right)$; LCMS m/z (ESI-QTOF, + ve): found 194.1199 ([M + $\left.2 \mathrm{H}]^{+}\right), \mathrm{C}_{11} \mathrm{H}_{16} \mathrm{~N}_{2} \mathrm{O}$ calculated 194.1414.

\section{(4-((4-ethylphenyl)amino)pyrrolidin-3-ol) (7d)}

Reddish oily; $38.5 \%$; IR (ATR) $v / \mathrm{cm}^{-1}$ : $3243.34(\mathrm{OH}), 2915(\mathrm{NH}$, stretch), $2845(\mathrm{CH}$, aromatic), 1518 (NH, bend), $1455\left(\mathrm{CH}_{2}\right) 1019$ (C-O), 820 (NH-Ar); ${ }^{1} \mathrm{H}-\mathrm{NMR}$ (400 MHz, CD $30 D$ ): $\delta 6.91(d, J=8.7 \mathrm{~Hz}, 2 \mathrm{H}), 6.59(d, J=8.7 \mathrm{~Hz}, 2 \mathrm{H})$, 3.79-3.72 (m, 2H), $3.70(d, J=5.9 \mathrm{~Hz}, 2 \mathrm{H}), 3.65$ $(d, J=3.7 \mathrm{~Hz}, 3 \mathrm{H}), 2.46(q, J=7.6 \mathrm{~Hz}, 2 \mathrm{H}), 1.98$ $(t, J=5.5 \mathrm{~Hz}, 1 \mathrm{H}), 1.12(t, J=7.5 \mathrm{~Hz}, 3 \mathrm{H}) ;{ }^{13} \mathrm{C} \mathrm{NMR}$ (100 MHz, CD $\left.{ }_{3} \mathrm{OD}\right): \delta 146.20$ (aromatic. C), 132.72 (aromatic, C), 128.08 (CH-Ar), $113.50(\mathrm{CH}-\mathrm{Ar}), 61.39$ $\left(\mathrm{CH}_{2} \mathrm{OH}\right), 60.28\left(\mathrm{CH}_{2}\right), 60.06\left(\mathrm{CH}_{2}\right), 54.30(\mathrm{CHNH})$, $44.66(\mathrm{CH}), 27.65\left(\mathrm{CH}_{2}\right), 15.32\left(\mathrm{CH}_{3}\right) ; \mathrm{LCMS} \mathrm{m} / \mathrm{z}$ (ESI-QTOF, + ve): found 222.1514 $\left([\mathrm{M}+2 \mathrm{H}]^{+}\right)$, $\mathrm{C}_{13} \mathrm{H}_{20} \mathrm{~N}_{2} \mathrm{O}$ calculated 222.1727.

\section{(4-((4-methoxyphenyl)amino)pyrrolidin-3-ol) (7e)}

Brown solid; $70.28 \%$; m.p. $84^{\circ} \mathrm{C}$. IR (ATR) v/cm-1: $3252(\mathrm{OH}), 2912(\mathrm{NH}$, stretch), $2830(\mathrm{CH}$, aromatic), $1508\left(\mathrm{NH}\right.$, bend), $1462\left(\mathrm{CH}_{2}\right), 1033(\mathrm{C}-\mathrm{O})$, 823 (NH-Ar); ${ }^{1} \mathrm{H}-\mathrm{NMR}$ (400 MHz, $\mathrm{CD}_{3} \mathrm{OD}$ ): $\delta 6.71$ (dd, $J=6.4,2.3 \mathrm{~Hz}, 2 \mathrm{H})$, 6.67-6.62 (m, 2H), 3.80-3.72 $(\mathrm{m}, 2 \mathrm{H}), 3.71-3.68(\mathrm{~m}, 2 \mathrm{H}), 3.67(\mathrm{~s}, 3 \mathrm{H}), 3.64(d, J$ $=5.5 \mathrm{~Hz}, 2 \mathrm{H}), 3.55(q, J=5.0 \mathrm{~Hz}, 1 \mathrm{H}), 1.97(q, \mathrm{~J}=$ $5.6 \mathrm{~Hz}, 1 \mathrm{H}) ;{ }^{13} \mathrm{C} \mathrm{NMR}\left(100 \mathrm{MHz}, \mathrm{CD}_{3} \mathrm{OD}\right): \delta 61.17$ $\left(\mathrm{CH}_{2}\right), 60.44\left(\mathrm{CH}_{2}\right), 60.14\left(\mathrm{CH}_{2}\right) 55.41(\mathrm{CH}), 54.85$ $(\mathrm{CH}), 44.66(\mathrm{CH})$; LCMS m/z (ESI-QTOF, + ve): found 224.1326 $\left([\mathrm{M}+2 \mathrm{H}]^{+}\right), \mathrm{C}_{12} \mathrm{H}_{18} \mathrm{~N}_{2} \mathrm{O}_{2}$ calculated 224.1519 .

\section{5-ethyl-4-(hydroxymethyl)pyrrolidin-3-ol (9a)}

Yellow oily; 99\%; IR (ATR) v/cm ${ }^{-1}: 3250$ $(\mathrm{OH}), 1549\left(\mathrm{NH}\right.$, bend), $1410\left(\mathrm{CH}_{2}\right), 1345\left(\mathrm{CH}_{3}\right)$, 1019 (C-O); ${ }^{1} \mathrm{H}-\mathrm{NMR}$ (400 MHz, CD $\mathrm{CD}_{3}$ ): $\delta$ 4.19$4.13(\mathrm{~m}, 1 \mathrm{H}), 3.62-3.49(\mathrm{~m}, 2 \mathrm{H}), 3.29-3.27(\mathrm{~m}, 1 \mathrm{H})$, 3.02-2.83 (m, 3H), 2.06-1.89 (m, 2H), $1.00(t, J=$ $7.5 \mathrm{~Hz}, 3 \mathrm{H}) ;{ }^{13} \mathrm{C} \mathrm{NMR}\left(100 \mathrm{MHz}, \mathrm{CD}_{3} \mathrm{OD}\right): \delta 73.75$ $(\mathrm{CHOH}), 62.57(\mathrm{CH}), 61.20\left(\mathrm{CH}_{2}\right), 55.32(\mathrm{CH}), 52.78$ $\left(\mathrm{CH}_{2} \mathrm{OH}\right), 27.19\left(\mathrm{CH}_{2}\right), 10.30\left(\mathrm{CH}_{3}\right)$; LCMS m/z (ESIQTOF, + ve): found 146.1161 $\left([\mathrm{M}+\mathrm{H}]^{+}\right), \mathrm{C}_{7} \mathrm{H}_{15} \mathrm{NO}_{2}$ calculated 146.1175 .

\section{4-(hydroxymethyl)-5-isopropylpyrrolidin-3-ol (9b)}

Yellow oily; 99\%; IR (ATR) v/cm ${ }^{-1}: 3293$ $(\mathrm{OH}), 2919(\mathrm{NH}$, stretch), $2850(\mathrm{CH}), 1506(\mathrm{NH}$, bend), $1408\left(\mathrm{CH}_{2}\right), 1019$ (C-O); ${ }^{1} \mathrm{H}-\mathrm{NMR}$ (400 $\left.\mathrm{MHz}, \mathrm{CD}_{3} \mathrm{OD}\right): \delta 4.27$ (s, $\left.1 \mathrm{H}\right), 3.61(d d, J=11.0$, $4.6 \mathrm{~Hz}, 1 \mathrm{H}), 3.45(d d, J=11.0,6.4 \mathrm{~Hz}, 1 \mathrm{H}), 3.10$ $(d, J=3.2 \mathrm{~Hz}, 2 \mathrm{H}), 2.89(d d, J=8.7,6.4 \mathrm{~Hz}, 1 \mathrm{H})$, 2.15-2.00 (m, 1H), 1.97-1.89 (m, 1H), $1.03(t, J=$ $6.9 \mathrm{~Hz}, 6 \mathrm{H}) ;{ }^{13} \mathrm{C}$ NMR (100 MHz, CD $\left.\mathrm{OD}\right): \delta 73.24$ $(\mathrm{CHOH}), 61.67\left(\mathrm{CH}_{2}\right), 52.73(\mathrm{CH}), 52.54\left(\mathrm{CH}_{2} \mathrm{OH}\right)$, $31.70(\mathrm{CH}), 18.80\left(\mathrm{CH}_{3}\right), 18.69\left(\mathrm{CH}_{3}\right) ; \mathrm{LCMS} \mathrm{m} / \mathrm{z}$ (ESI-QTOF, + ve): found 160.1315 $\left([\mathrm{M}+\mathrm{H}]^{+}\right)$, $\mathrm{C}_{8} \mathrm{H}_{17} \mathrm{NO}_{2}$ calculated 160.1332 . 


\section{4-(hydroxymethyl)-5-(p-tolyl)pyrrolidin-3-ol (9c)}

Yellow solid; $68.27 \%$; m.p. exceed $270^{\circ} \mathrm{C}$ decomposed. IR (ATR) v/cm $\mathrm{cm}^{-1}: 3676(\mathrm{OH}), 3565$ $(\mathrm{NH}$, stretch), $2850(\mathrm{CH}$, aromatic), $1574(\mathrm{NH}$, bend), $1420\left(\mathrm{CH}_{2}\right), 843\left(\mathrm{Ar}-\mathrm{CH}_{3}\right),{ }^{1} \mathrm{H}-\mathrm{NMR}(400$ $\left.\mathrm{MHz}, \mathrm{CD}_{3} \mathrm{OD}\right): \delta 7.28(d, J=8.2 \mathrm{~Hz}, 2 \mathrm{H}), 7.13(d, J$ $=7.8 \mathrm{~Hz}, 2 \mathrm{H}), 4.28(\mathrm{~s}, 1 \mathrm{H}), 3.72(d, J=7.8 \mathrm{~Hz}, 1 \mathrm{H})$, $3.62(\mathrm{~s}, 1 \mathrm{H}), 3.49(\mathrm{~s}, 1 \mathrm{H}), 2.97(d, J=13.3 \mathrm{~Hz}, 2 \mathrm{H})$, $2.30(d, J=4.6 \mathrm{~Hz}, 3 \mathrm{H}), 2.11-2.00(1 \mathrm{H}) ;{ }^{13} \mathrm{C}$ NMR (100 MHz, CD 3 OD ) $\delta 140.71$ (aromatic. C), 135.60 (aromatic. C), $128.88(\mathrm{CH}-\mathrm{Ar}), 126.56(\mathrm{CH}-\mathrm{Ar}), 72.10$ $(\mathrm{CHOH}), 66.90(\mathrm{CH}), 57.00\left(\mathrm{CH}_{2}\right), 56.20(\mathrm{CHOH})$, $48.31(\mathrm{CH}), 21.3\left(\mathrm{CH}_{3}\right)$; LCMS $\mathrm{m} / \mathrm{z}$ (ESI-QTOF, + ve): found $208.1308\left([\mathrm{M}+\mathrm{H}]^{+}\right), \mathrm{C}_{12} \mathrm{H}_{17} \mathrm{NO}_{2}$ calculated 208.1332 .

\section{4-(4-hydroxy-3-(hydroxymethyl)pyrrolidin-2-yl) benzonitrile (9d)}

Brown oily; 67.84\%; IR (ATR) v/cm ${ }^{-1}: 3319$ $(\mathrm{OH}), 2915(\mathrm{NH}$, stretch), $2848(\mathrm{CH}$, aromatic), $1636.93\left(\mathrm{NH}\right.$, bend), $1420\left(\mathrm{CH}_{2}\right), 1018(\mathrm{C}-\mathrm{O}), 606$ (Ar-CN); ${ }^{1} \mathrm{H}-\mathrm{NMR}$ (400 MHz, CD $\left.{ }_{3} \mathrm{OD}\right): \delta 7.29$ (d, J $=8.2 \mathrm{~Hz}, 2 \mathrm{H}), 7.14(d, J=7.8 \mathrm{~Hz}, 2 \mathrm{H}), 4.35-4.25$ $(\mathrm{m}, 1 \mathrm{H}), 3.78(d, J=8.2 \mathrm{~Hz}, 1 \mathrm{H}), 3.64(d d, J=$ 11.2, $4.8 \mathrm{~Hz}, 1 \mathrm{H}), 3.48$ (q, $J=5.6 \mathrm{~Hz}, 1 \mathrm{H}), 2.99$ $(t, J=5.3 \mathrm{~Hz}, 2 \mathrm{H}), 2.09(\mathrm{~s}, 1 \mathrm{H}) ;{ }^{13} \mathrm{C}$ NMR $(100$ $\mathrm{MHz}, \mathrm{CD}_{3} \mathrm{OD}$ ): $\delta 138.41$ (aromatic. C) $137.18(\mathrm{CN})$ $128.98 \mathrm{CH}-\mathrm{Ar}$ ), 127.13 (CH-Ar), 126.46 (aromatic. C) $74.53(\mathrm{CHOH}), 64.57(\mathrm{CH}), 60.40\left(\mathrm{CH}_{2}\right), 58.35$ $(\mathrm{CH}), 54.21\left(\mathrm{CH}_{2} \mathrm{OH}\right)$; LCMS m/z (ESI-QTOF, + ve): found $222.1350\left([\mathrm{M}+4 \mathrm{H}]^{+}\right), \mathrm{C}_{12} \mathrm{H}_{14} \mathrm{~N}_{2} \mathrm{O}_{2}$ calculated 222.1363

\section{4-(hydroxymethyl)-5-(4-methoxyphenyl)-1-} methylpyrrolidin-3-ol (9e)

Brown oily; 32.33\%; IR (ATR) v/cm ${ }^{-1}: 3319$ $(\mathrm{OH}), 1560$ (NH, bend), $1408\left(\mathrm{CH}_{2}\right), 1026(\mathrm{C}-\mathrm{O}), 628$ (Ar-OMe); ${ }^{1} \mathrm{H}-\mathrm{NMR}$ (400 MHz, $\left.\mathrm{CD}_{3} \mathrm{OD}\right): \delta 7.42$ (d, $J=9.1 \mathrm{~Hz}, 2 \mathrm{H}), 6.92(d, J=9.1 \mathrm{~Hz}, 2 \mathrm{H}), 4.61-4.52$ $(\mathrm{m}, 1 \mathrm{H}), 4.13(d, J=10.1 \mathrm{~Hz}, 1 \mathrm{H}), 3.78(\mathrm{~s}, 3 \mathrm{H})$, $3.55(d d, J=11.0,8.7 \mathrm{~Hz}, 1 \mathrm{H}), 3.44(d, J=11.0 \mathrm{~Hz}$,
$1 \mathrm{H}), 3.14(q, \mathrm{~J}=5.3 \mathrm{~Hz}, 1 \mathrm{H}), 3.05(d d, J=11.0,4.1$ $\mathrm{Hz}, 1 \mathrm{H}), 2.76-2.64(\mathrm{~m}, 1 \mathrm{H}), 2.55(\mathrm{~s}, 3 \mathrm{H}) ;{ }^{13} \mathrm{C}$ NMR (100 MHz, CD $\left.{ }_{3} \mathrm{OD}\right): \delta 178.49$ (aromatic. C), 160.19 (aromatic. C), 130.54 (CH-Ar), $113.68(\mathrm{CH}-\mathrm{Ar})$, $72.50(\mathrm{CHOH}), 69.38(\mathrm{CH}), 62.88(\mathrm{CHOH}), 58.20$ $\left(\mathrm{CH}_{2} \mathrm{OH}\right), 54.89\left(\mathrm{OCH}_{3}\right), 48.68(\mathrm{CH}), 39.10\left(\mathrm{CH}_{3}-\mathrm{N}\right)$; LCMS $m / z$ (ESI-QTOF, + ve): found 238.1499 ([M + $\left.\mathrm{H}]^{+}\right), \mathrm{C}_{13} \mathrm{H}_{19} \mathrm{NO}_{3}$ calculated 238.1438.

\section{Determination of alpha-glucosidase inhibition activity}

The alpha-glucosidase enzyme inhibitory activity assay was adopted from the method described by Lee et al., with slight modification ${ }^{14}$. The inhibition rate of alpha glucosidase was determined at $37^{\circ} \mathrm{C}$ in phosphate buffer solution ( $\mathrm{pH} \mathrm{6.5).} \mathrm{The}$ reaction mixtures containing $10 \mu$ l of sample, $20 \mu \mathrm{l}$ of alpha glucosidase enzyme, $20 \mu$ of water and 40 $\mu \mathrm{l}$ of buffer solution were mixed in microtiter plate. The mixtures were pre-incubated at $37^{\circ} \mathrm{C}$ for 10 minutes. Then, $10 \mu$ l of substrate in buffer solution were added, and the incubation time was prolonged to an additional 30 minutes. The absorption at 405 $\mathrm{nm}$ was measured instantly after 30 min of incubation with microplate spectrophotometer and the inhibition activity was calculated by the equation described by Lee et al., ${ }^{14}$.

\section{RESULTS AND DISCUSSION}

\section{Synthesis of pyrrolidine-based iminosugar derivatives}

The synthetic strategy used in this study is outlined in Scheme 1. The pyrrolidine based iminosugar derivatives were synthesized in three or four steps linear synthetic route (Scheme 1) starting with the multicomponent reaction (MCR), amination, reduction of olefinic bond followed by simultaneous reductions of both the ester and amide (for compounds 7c-e using 4 steps strategy) (Scheme 1).

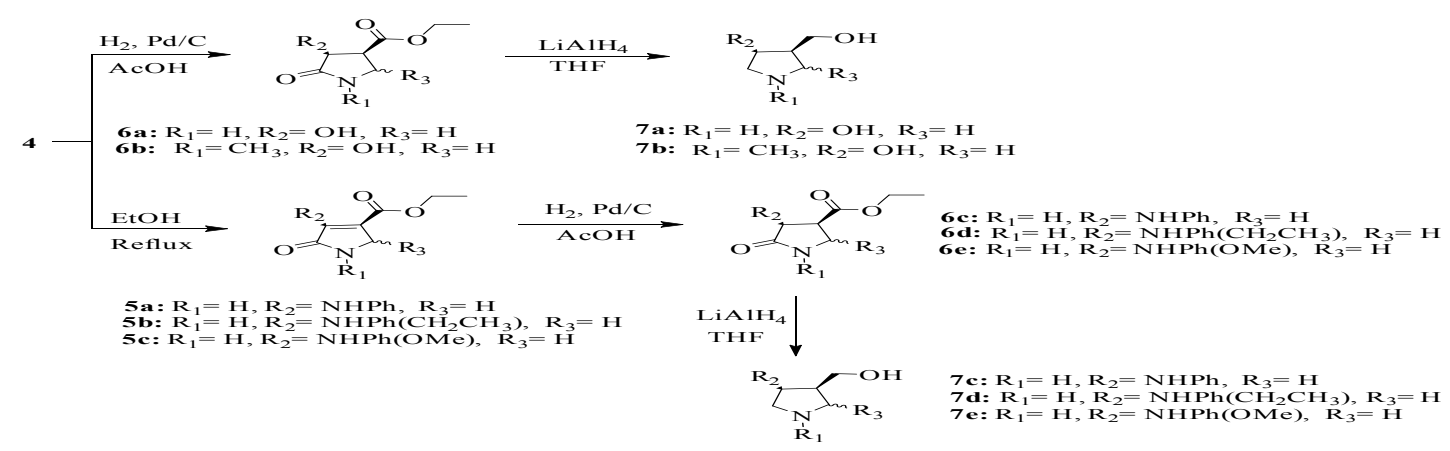


According to Mohammat et al., the 2,3dioxopyrolidine skeleton 4 can be obtained through a multicomponent reaction by reacting sodium diethyl oxaloacetate with the same molar concentrations of aldehydes 3 and amines 2 at reflux condition in moderate yields7 (Scheme 2).

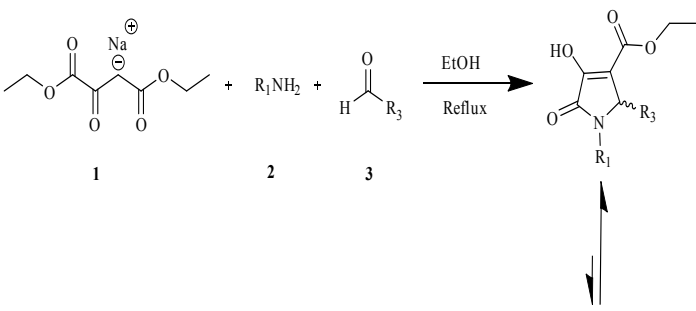

$$
\begin{aligned}
& \sum_{\substack{\mathrm{N} \\
1 \\
\mathrm{R}_{1}}}^{0} \ln _{\mathrm{R}_{3}} \\
& \text { 4a: } \mathrm{R}_{1}=\mathrm{H}, \mathrm{R}_{3}=\mathrm{H} \\
& \text { 4b: } \mathrm{R}_{1}=\mathrm{CH}_{3}, \mathrm{R}_{3}=\mathrm{H} \\
& \text { 4c: } \mathrm{R}_{1}=\mathrm{H}, \mathrm{R}_{3}=\mathrm{CH}_{2} \mathrm{CH}_{3} \\
& \text { 4d: } \mathrm{R}_{1}=\mathrm{H}, \mathrm{R}_{3}=\mathrm{CH}\left(\mathrm{CH}_{3}\right)_{2} \\
& \text { 4e: } \mathrm{R}_{1}=\mathrm{H}, \mathrm{R}_{3}=\mathrm{Ph}\left(\mathrm{CH}_{3}\right) \\
& \text { 4f: } \mathrm{R}_{1}=\mathrm{H}, \mathrm{R}_{3}=\mathrm{Ph}(\mathrm{CN})
\end{aligned}
$$

\section{Scheme 2. Synthesis of 2,3-dioxopyrrolidines via a multicomponent reaction}

The crude product was then acidified and filtered to give the desired products, 4a-k. However, for the synthesis of compound $4 \mathrm{a}$ an excess of amount of ammonia is required ${ }^{15}$. The amination of compound 2,3-dioxopyrrolidines 4 a of the keto group at C-3 gave derivatives of different 4-hydroxy-5-oxo2,5-dihydro-1 $\mathrm{H}$-pyrrole-3-carboxylic acid esters 5a$\mathrm{C}^{16}$. These attempts were refluxed in ethanol for 12 and $24 \mathrm{~h}$ but the yield obtained were low (21-26\%). Jourdan et al., reported similar observation during the amination of 4-hydroxy-5-oxo-2,5-dihydro- $1 \mathrm{H}$ pyrrole-3-carboxylic acid esters molecule ${ }^{17,16,18}$. The formic acid was added to favor the $4 a$ in keto form ${ }^{19}$, however the yields $5 \mathrm{a}-\mathrm{c}$ were similar .
Subsequently, compounds $5 \mathrm{a}-\mathrm{c}$ were treated with acetic acid in the presence of $\mathrm{Pd} / \mathrm{C}$ catalyst which underwent high diastereoselective hydrogenation at the olefenic bond of the pyrrolidine skeleton yielding $6 a-e$ as cis-configured isomers ${ }^{8}$. Reductions of the enolic tautomers $4 \mathrm{a}$ and $4 \mathrm{~b}$ by hydrogenation were performed under neutral or acidic conditions in ethanol for 3 hours. The mechanism of the catalytic reduction of these compounds was proposed by Mohammat $2015^{8}$. This strategy furnished the cis-hydroxy esters $6 \mathrm{a}$ and $6 \mathrm{~b}$ in with moderate to high yields $58 \%$ and $98 \%$, respectively 8 . Similarly, stereoselective reduction of enamine tautomers $5 \mathrm{a}-\mathrm{c}$ via syn hydrogenation was also performed under neutral condition for $24 \mathrm{~h}$ but the yields obtained were low (31-36\%). Adding acetic acid as the catalyst to the reaction condition reduced the reaction time, but the yields were not improved $^{20,21,22,23}$.

The catalytic hydrogenation of $5 \mathrm{a}-\mathrm{c}$ is formed via the chelation ring formation as proposed by Harada and Matsumoto ${ }^{24}$. The six-membered chelated ring structure with the catalyst is present in the intermediate, the hydrogen atom preferentially attacked from the less hindered side to afford compounds $6 \mathrm{c}-\mathrm{e} 24$. The reduction of enamine was found to be low in yield under acidic and neutral conditions due to the presence of nitrogen atom of the amine derivatives which poisoned the palladium catalyst and degraded the selectivity of the catalyst ${ }^{25}$. These reactions were caused by the electron donor of $\mathrm{N}-\mathrm{H}$ group of an amine derivatives, which has the capability to compete for coordination at the catalytic site ${ }^{25}$. Similar observation reported by Xie et al., during hydrogenation of enamines and imines26. Compounds 7a-e were obtained by reduction of amide and ester via excess $\mathrm{LiAlH}_{4}$ to give the target molecule ${ }^{27}$.

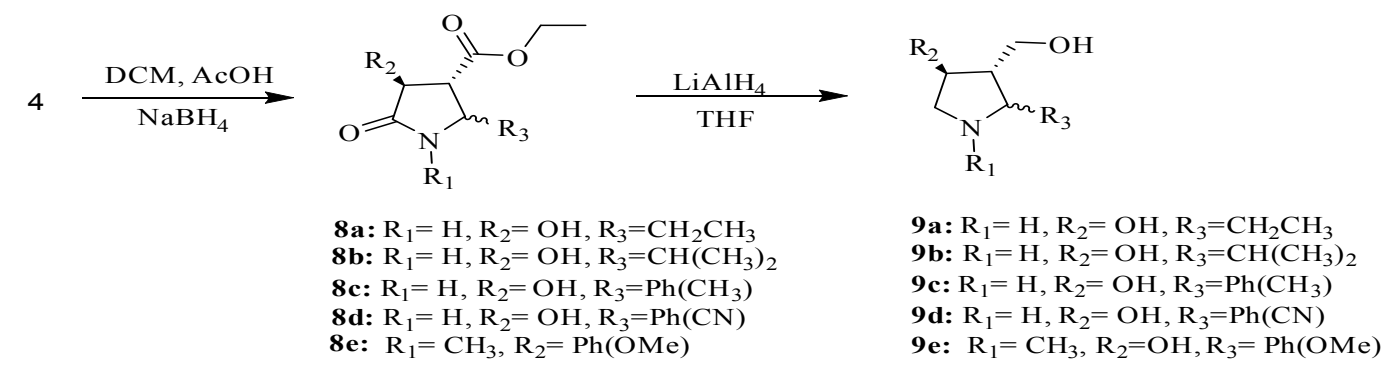

Scheme 3. Synthesis of the Pyrrolidine-based iminosugars (9a-e) 
Alternatively, 2,3-dioxo-4-carboxypyrrolidines $(4 \mathrm{c}-\mathrm{g})$ underwent stereoselective reduction using $\mathrm{NaBH}_{4} / \mathrm{AcOH}$ to specifically yield $8 \mathrm{a}-\mathrm{e}$ as trans-configured analogues in good yields. The mechanism and steric effect in this type of reduction reaction was proposed by Mohammat 2015 as shown in Fig. $2^{8}$. It was predicted throughout the reaction, compounds 4 tautomerized into both the enol and keto forms; however the keto analogues are more stable due to reaction being catalyzed by an acid. In terms of diastereoselection, the bulky C-5 substituent noticeably contributed towards the steric factor and this gave only the thermodynamicallystable trans product $(8 \mathrm{a}-\mathrm{e})^{8}$. Therefore, the hydride was transfered from the less hindered part of $P$, away from the $\mathrm{C}-5$ substituent forcing the hydroxy group to be in trans-position to that of the ethyl ester ${ }^{8}$. Then, the amide and ester functionalities of compounds $8 a-e$ were reduced further with an excess $\mathrm{LiAlH}_{4}$ to give compounds $9 \mathrm{a}$-e in moderate to high yields.

(P)

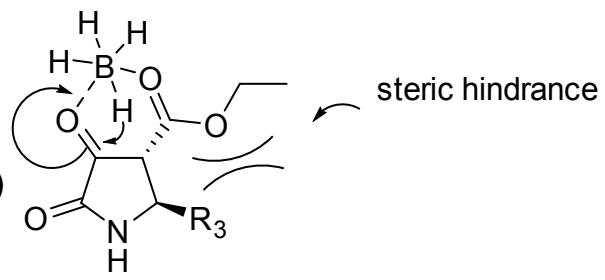

Fig. 2. Mechanistic reduction

Biological activity of pyrrolidine-based iminosugar derivatives

Ten iminosugars and their respective intermediates were synthesized to evaluate their -glucosidase inhibitory activity in comparison with deoxynojirimycin (DNJ) as the positive control, as shown in Table 1. The inhibition percentages against glucosidase of these were tested on two different concentrations at $1.0 \mathrm{mM}$ and $5.0 \mathrm{mM}$. The inhibition percentages in $1.0 \mathrm{mM}$ and $5.0 \mathrm{mM}$ of DNJ were found to be $59.38 \pm 1.22$ and $80.75 \pm 0.38$, respectively and it was regarded as a positive control. In general, the present or increase in the inhibition activity is closely associated with the aryl subtituents bearing either electron donating groups (-OMe and $\mathrm{CH}_{3}$ ) or an electron withdrawing group $(-\mathrm{CN})$. Among the ten synthesized iminosugars, $7 \mathrm{e}$ gave the highest inhibition of $67.5 \pm 0.5$ at $1.0 \mathrm{mM}$ followed by $9 \mathrm{c}$ with $17.9 \pm 3.5$ and $7 \mathrm{c}$ with $4.3 \pm 0.7$. Compound $7 \mathrm{e}$ gave better inhibition activity at $1.0 \mathrm{mM}$ concentration towards glucosidase as compared to DNJ. The presence of methoxy-containing arylamine at C-3 position somehow contributes towards the formation of iminosugars as potential glucosidase inhibitors. Further modification at the pyrrolidine skeleton is thus to enhanced the bio activity of the imino sugar while maintaining the presence of the p-methoxy arylamine at the C-3 position.

Table 1: Alpha-Glucosidase inhibition studies of synthesize compunds

\begin{tabular}{|c|c|c|}
\hline \multirow[t]{2}{*}{ Iminosugars } & \multicolumn{2}{|c|}{ Alpha-Glucosidase Inhibition (\%) } \\
\hline & $1.0 \mathrm{mM}$ & $5.0 \mathrm{mM}$ \\
\hline $5 b$ & NT & NT \\
\hline $6 e$ & NT & NT \\
\hline $7 a$ & $\mathrm{NI}$ & $\mathrm{NI}$ \\
\hline $7 \mathrm{~b}$ & $\mathrm{NI}$ & $\mathrm{NI}$ \\
\hline $7 \mathrm{c}$ & $4.3 \pm 0.7$ & $16.2 \pm 1.1$ \\
\hline $7 d$ & $\mathrm{NI}$ & $5.7 \pm 0.7$ \\
\hline $7 e$ & $67.5 \pm 0.5$ & $\mathrm{NI}$ \\
\hline $9 a$ & $\mathrm{NI}$ & $\mathrm{NI}$ \\
\hline $9 b$ & $\mathrm{NI}$ & $\mathrm{NI}$ \\
\hline 9c & $17.9 \pm 3.5$ & $80.9 \pm 0.3$ \\
\hline $9 d$ & $\mathrm{NI}$ & $16.5 \pm 1.8$ \\
\hline $9 \mathrm{e}$ & $\mathrm{NI}$ & $\mathrm{NI}$ \\
\hline DNJ & $59.38 \pm 1.22$ & $80.75 \pm 0.38$ \\
\hline
\end{tabular}

$\%$ Inhibition determined at $1.0 \mathrm{mM}$ and $5.0 \mathrm{mM}$ concentration of compound

NI: No Inhibition

NT: Not Tested

\section{CONCLUSION}

In conclusion, ten pyrrolidine-based iminosugars were synthesized in three or four steps utilizing MCR as the key step. One compound, 7e (4-((4-methoxyphenyl)amino)pyrrolidin-3-ol) demonstrated a strong potent inhibitory activity at $1.0 \mathrm{mM}$ concentration against alpha-glucosidase test. In addition, the presence of a methoxy group at C-3 plays an important role in the inhibition and that further structural variations at the nitrogen atom of the skeleton using different amine derivatives could improve the alpha glucosidase activity.

\section{ACKNOWLEDGMENT}

The authors wish to thank the Institute of Science (IOS), UiTM Shah Alam, Malaysia for its generous support and the Ministry of Education (MOE), Malaysia for the financial support under the Fundamental Research Grant Scheme (600-IRMI/ FRGS 5/3 (109/2019).

\section{Conflict of Interests}

The authors declare that there is no conflict of interests related to the publication of this paper. 


\section{REFFERENCES}

1. Prasad, P.; Surajit, M.; Sudhir, K. T.; Santosh, A., Eur. J. Nutr., 2015. 2. Sivaprasad, K.; Sujatha, S.; Srinivas, U.; Jaya, S.
A.; Shubham D.; Hasitha, S. A.; Yogeeswari, P.; Dilep K. S.; Bathini N. B.; Krishna, S. E., Bioorg., Med. Chem. Lett., 2017, 27, 2818-2823.

3. Clifford, J. B.; Caroline, D., Br J Cardiol., 2003, 10, 128-36.

4. Lucassen, P.; Rutten, G.; Lucassen, C. V. W.; Rutten, P.; Van, G. W. C., Cochrane Database Syst. Rev., 2005, 2.

5. Erica, C. S.; Nathália, C. G. Y.; Alcindo, A. D. S.; Fernando, C. R., Synthesis., 2017, 49, 4869-4875.

6. Doddi, V. R.; Yashwant D. V., European J. Org. Chem., 2007, 33, 5583-5589.

7. Mohammat, M. F.; Shaameri, Z.; Hamzah, A. S., Molecules., 2009, 14(1), 250-256.

8. Mohammat, M. F.; Mansor, N.S.; Shaameri, Z.; Hamzah, A. S., J. Korean Chem. Soc., 2015, 59(1), 31-35.

9. Zhang, E.; Bai, P.Y.; Sun, W.;Wang, S.; Wang, M. M.; Xu, S. M., Carbohydr. Res., 2016, 434, 33-36.

10. Kotkar, S. P.; Chavan, V. B.; Sudalai, A., Org. Lett., 2007, 9(6) 1001-1004.

11. Masakazu, S.; Zhangyong, H.; Liang, P. H.; Stephen, M. D.; Lisa, J. W. H.; William, A. G.; Chi-Huey., J. Am. Chem. Soc., 2007, 6, 14811-14817.

12. En-Lun, T.; Sih-Yu, C.; Ming-Hsun, Y.; ShihChi, W.; Ting-Ren, R. C., Wei-Chieh, C., Bioorg. Med. Chem., 2008, 16(24), 1019810204.

13. Shaameri, Z.; Ali, S. H. S.; Mohamat, M. F.; Yamin, B. M.B.; Hamzah, A. S., J. Heterocycl. Chem., 2009, 0, 1208-1212.
14. Lee, S.; Lin, H.; Chen, C., Phytochemistry., 2008, 69, 2347-2353.

15. Metten, B.; Kostermans, M.; Baelen, G. V.; Smet, M.; Dehaen, W., Tetrahedron., 2006, 62(25), 6018-6028.

16. Southwick, P. L.; Hofmann, G.H., J. Org. Chem., 1963, 28(5), 1332-1336.

17. Jourdan, F.; Kaiser, J. T.; Lowe, D. J., Synth. Commun., 2006, 35, 2453-2466.

18. Augustin, M.; Jeschke, P., J. prak. Chem., 1987, 329, 599-606.

19. Madhav, R.; Richard, F. D.; Southwick, P. L., J. Heterocycl. Chem., 1973, 10, 25-28.

20. Ikemoto, N.; Tellers, D.M.; Dreher, S.D.; Liu, J; Huang, A.; Rivera, N. R.; Njolito, E.; Hsiao, Y.; Williams, J. C.; Williams, J.M.; Armstrong, J. D.; Yongkui, S.; Mathre, D. J.; Grabowski, E. J. J.; Tillyere, R. D., J. Am. Chem. Soc., 2004, 126(10), 3048-3049.

21. Richard F. B.; Mark D. B.; Durstl, H. D., J. Am. Chem. Soc., 1970, 3(1), 1968-1970.

22. Smaliy, R. V.; Aleksandra A. C.; Nataliya A. S.; Sergey A. Y.; Aleksandr A. Y.; Aleksandr I. L.; Alina O. G.; Aleksandr N. K., J. Fluor. Chem., 2015, 180, 257-264.

23. Tungler, B. A.; Tarnai, T.; Hegediis, L.; Fodor, K.; Mathe, T., Platinum Metals Rev., 1998, 42(3), 108-115.

24. Mitsuru, F.;Tadashi, O.; Yoshihide, N.;Yuriko, T., Chem. Pharm. Bull., 1979, 27(9), 2223-2225.

25. Marcazzan, P; Patrick, B. O.; James, B. R. Organometallics., 2003, 22(6), 1177-1179.

26. Xie, J. H.; Zhu, S. F.; Zhou, Q. L., Chem. Rev., 2011, 111(3), 1713-1760.

27. Goti, A.; Cicchi, S.; Cacciarini, M.; Cardona, F.; Fedi, V.; Brandi, A., European J. Org. Chem., 2000, 21, 3633-3645. 